\title{
LVII. Value of different kinds of vegetable food, based upon the amount of nitrogen
}

\section{E.N. Horsford M.A.}

To cite this article: E.N. Horsford M.A. (1846) LVII. Value of different kinds of vegetable food, based upon the amount of nitrogen, Philosophical Magazine Series 3, 29:195, 365-397, DOI: $10.1080 / 14786444608645526$

To link to this article: http://dx.doi.org/10.1080/14786444608645526

曲 Published online: 30 Apr 2009.

Submit your article to this journal $₫$

Џ Article views: 2

Q View related articles $\square$

4 Citing articles: 1 View citing articles ๘ 
days they are hardly perceptible, though they never cease altogether. On some days they are violent, if I may be allowed the expression. The needle does not take a sudden start and return as when influenced by lightning, but moves gradually without oscillation to some fixed point, from which it will return sometimes in two minutes and sometimes in ten or fifteen minutes. An extended series of observations will be necessary before any deductions can be safely made. If the wires should be separated by a slight interval during a thunder storm, doubtless electrical sparks would be visible. During heavy storms, a flash of lightning twenty miles distant from the wires of Morse's telegraph will induce electricity in the wire sufficient to operate the magnets and work the telegraph, sometimes recording several signals. A flash of lightning in Baltimore, forty miles distant from this place, will operate the magnet at this end of the line.

Washington, D. C.

LVII. Value of different kinds of Vegetable Food, based upon the amount of Nitrogen. By E. N. Horsford, M.A.*

SINCE Gay-Lussac's discovery of nitrogen in the seeds of $\$$ plants, the conception of animal nutrition has been assuming a more and more definite character.

Already have the principal proximate ingredients of meal, by taking advantage of its physical properties, been separated from each other. Gluten, albumen and legumine, starch, gum, sugar, dextrine and woody-fibre, have been made known, and their physical as well as some of their chemical properties $\dagger$ studied. Their more accurate chemical constitution was reserved to a later period, when it was found that they might be arranged in two classes, those containing nitrogen and those containing no nitrogen; and the interesting discovery was made, that the former, as well as the latter, are among themselves identical in composition.

It is well known that labourers supplied only with food containing no nitrogen, become incapable of executing their tasks: and further, that the corporeal system, even without labour, cannot be sustained upon such food. The discovery of the near identity in chemical composition between vegetable albumen, fibrine and caseine, and the corresponding bodies

- Communicated by the Author.

+ Fr. Marcet found gluten consisting of 55.7 per cent. carbon, 22 per cent. oxygen, $7 \cdot 8$ per cent. hydrogen, and $14 \cdot 5$ per cent. nitrogen. $-A n n$. de Ch. et de Phys. xxxvi. 27. 
found in the animal kingdom, gave the above fact its explanation.

The food must contain an ingredient suited to replace the nnimal matter consumed.

This being known, and the quantitative relation of the several elements of the nitrogenous compounds being also known, an estimate of the value of given kinds of food becomes, in the hands of the chemist, a problem of comparatively easy solution.

The following investigation, undertaken at the suggestion and under the direction of Prof. von Liebig in the Giessen laboratory, had for its object the determination of the relative values of the different kinds of vegetable food. These values are threefold.

The various forms of food derived from grains, herbage and roots, furnish -1, bodies containing nitrogen; 2 , bodies destitute of nitrogen; and 3, inorganic salts, all of which are serviceable in the animal œconony.

The nitrogenous bodies, from their solution in the blood, form the tissues-the actual organism. The bodies wanting nitrogen contribute, by their more or less perfect combustion, to the warmth of the animal body; and the salts of the alkalies and alkaline earths serve in building up the osseous framework, beside constituting an essential part of every organ of the animal system.

Their values for the latter purpose are in general in proportion to the phosphates their ashes contain.

Their values for the second purpose may be considered in general as in the inverted relation of their values for the first, or inversely proportioned to the per-centage of nitrogen.

Their values for the first purpose, that of ministering to the support and growth of organic tissues, have been the specific object of the hereafter enumerated determinations.

Boussingault, to whom the agriculturist is so largely indebted for investigations bearing upon the interests of husbandry, has not left this field untrodden. It was thought, however, that the worth of his table of nutrition could lose nothing by a series of carefully conducted analyses, having the same object in view. It was moreover conceived, that in substances containing so small a per-centage of nitrogen as grains and roots generally, the method of Messrs. Varrentrapp and Will for determining nitrogen would give more accurate results than that of Dumas employed by Boussingault. 'The analyses hereafter given of the same substance, rarely varied from each other more than one-tenth of one per cent : and yet in general the determinations which follow, and those of similar 
substances made by the distinguished French chemist, differ no further from each other than might be expected from productions of the same vegetable variety grown on different soils.

Buckwheat (Polygonum Fagopyrum) constitutes an exception to this remark. In the table of analytical results* this grain has a nitrogen per-centage of $2 \cdot 40$, while two ordinary varieties of wheat (Triticum vulgare) have 2.33 and 2.90 per cent. Buckwheat meal from Vienna gave, as shown below, 1.08 per cent. Buckwheat grains (Polygonum tartaricum) from the experimental field of Hohenheim Agricultural Institute, gave 1.56 per cent. of nitrogen, while the analysis of three superior varieties of wheat grown in the same field, gave respectively $2 \cdot 59,2 \cdot 68$ and 2.69 per cent. This species of buckwheat was further found to contain 22.66 per cent. of woody fibre.

The equivalent value of buckwheat, according to Boussin. gault, wheat being 100, is 108. The following analyses gave for its equivalent value 170 ; for that of the Vienna buckwheat meal 24.5 .

For the following investigation, the meals, table-peas and beans and lentils, were procured by Prof. von Liebig from Vienna. The grains, with the exception of rice and Triticum monococcum, were furnished from the Agriculturai Institute at Hohenheim in the kingdom of Würtemberg, in reply to an order for the most approved varieties of Cerealia cultivated in Europe. The roots were from Giessen.

The several grains, meals and roots, in their market condition, were dried in a water-bath at $100^{\circ} \mathrm{C}$. The potatoes, beets, carrots and turnips, were cut into thin shavings with the least delay, weighed between watch-glasses and placed in the water-bath.

The combustions for carbon and hydrogen were made with oxide of copper, a mixture of chlorate of potassa and oxide having been placed at the extreme end of the combustiontube.

It was found difficult to reduce the woody fibre of the oats, barley and buckwheat, to the requisite fineness for a complete combustion. Where the difficulty could not readily be overcome, in addition to the chlorate of potassa at the end of the tube, it was found well, in filling with mixed substances and oxide of copper, to add, at intervals of an inch and a half, a small quantity of finely pulverized and thoroughly mixed oxide of copper and chlorate of potassa. The successive evolutions of oxygen in this case thoroughly reoxidized any por-

* Boussingault's Landswirthschaft, Germ. ed., p. 294. 
tions of copper reduced in the progress of combustion, and secured the most satisfactory results.

Difficulty presented itself also in the combustion of the beets and other roots, arising from the extreme compactness of the substance when dried. It was however overcome by the method already mentioned.

The per-centage of woody fibre was determined in the following manner. Grains, such as had been analysed, were digested upon a sand-bath, several weeks, in diluted hydrochloric acid,-one part of acid to a thousand of water. At intervals of from eight to ten days the fluid was poured off, and the digestion resumed with diluted acid, as before. After several weeks, the woody fibre not appearing wholly freed from other substances, an equally dilute solution of caustic potassa was employed, and the digestion therewith continued. At the end of two months, the woody fibre of the oats, barley and buckwheat, were poured upon filters, thoroughly washed with distilled water, and dried at $100^{\circ} \mathrm{C}$. The hulls of the peas and beans were treated with diluted caustic potassa alone, and similarly determined.

To express in 100 parts the results of analyses, the carbon, hydrogen, oxygen and sulphur of the nitrogenous ingredients were estimated from the per-centage of nitrogen *. The carbon and hydrogen so estimated, deducted from the whole per-centage of carbon and hydrogen, gave what belonged to the starch, gum, sugar, \&c. The oxygen of the latter was estimated from the carbon by the formula $\mathrm{C}_{12} \mathrm{H}_{10} \mathrm{O}_{10}+$.

- Mulder's analysis of coagulated albumen, Scheerer and Jones's analyses of legumine and gluten (Ann. de Ch. und Pharm. xxxix. 360), and Heldt's analysis of the gluten of rye (Ann. de Ch. und Pharm. xlv. 198), differ so little from each other, that a single formula has been constructed upon Mulder's analysis of coagulated albumen, and, with a little modification, employed in determining the elements of the nitrogenous ingredients of all the substances analysed. His per-centage of oxygen was reduced by the per-centage of sulphur, which, according to Dr. Rüling ( $A n n$. de $C h$. und Pharm. lviii. $3 \mathrm{Heft}$.), for gluten $=1.14$ per cent. and for legumine 0.50 per cent. The phosphorus has been neglected, as no accurate determinations of the per-centage of this element has been made. Mucine, discovered by Berzelius, is included in the estimate for the nitrogenous ingredients, since analyses of albumen and of ghuten, with and without mucine, corre. spond with each other.

+ The following list of the principal organic bodies containing no nitrogen, and present in the substances analysed, and their adjoined constitution, will justify the method of calculation :-

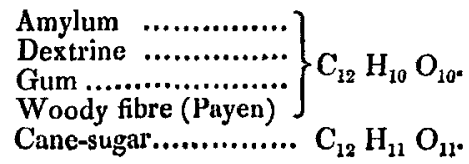


No. 1. Wheat-flour, from Vienna.

I. 1.0883 gramme lost at $100^{\circ}$ C. $0.9378 \mathrm{grm}$.

II. $1.3090 \mathrm{grm}$. of the flour dried at $100^{\circ} \mathrm{C}$. left, after incineration, $0.0091 \mathrm{grm}$.

III. $0.8620 \mathrm{grm}$. of the same left $0.0061 \mathrm{grm}$. ashes.

IV. 0.3805 grm. burned with oxide of copper, gave 0.6370 grm. of carbonic acid and $0.2186 \mathrm{grm}$. of water.

V. $0.4190 \mathrm{gr}$ gave $0.7020 \mathrm{gr}$. $\mathrm{CO}^{2}$ and $0.2550 \mathrm{gr}$. HO.

VI. $0.3671 \mathrm{gr}$. gave $0.6189 \mathrm{gr}$. $\mathrm{CO}^{2}$ and $0.2374 \mathrm{gr}$. HO.

VII. 0.8078 gr. gave, by Varrentrapp and Will's method for determining nitrogen, $0.3925 \mathrm{gr}$. ammonio-chloride of platinum. tinum.

VIII. $0.8168 \mathrm{gr}$. gave $0.3893 \mathrm{gr}$, ammonio-chloride of pla-

These determinations correspond, expressed in 100 parts, to-

I.

Carbon ... $\quad 4.5 \cdot 66$

Hydrogen $\quad 6.38$

Nitrogen . $\quad 3.04$

Ashes ... 0.69

Water ... 13.83
II.

$45 \cdot 69$

$6 \cdot 76$

$2 \cdot 99$

0.70

Estimated in 100 parts, according to the constitution of the ingredients present, the above determinations give the following numbers :-

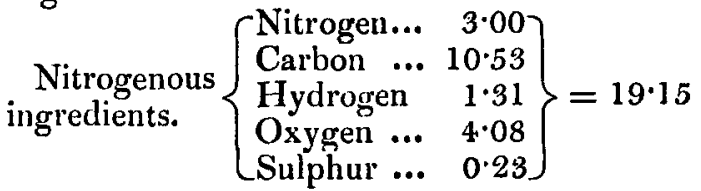

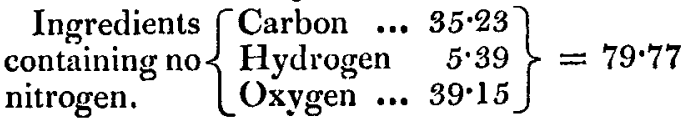

$$
\begin{aligned}
& \text { Ashes................ }=\frac{0.70}{99.62}
\end{aligned}
$$

No. 2. Wheat-four from Vienna.

I. 3.6365 grms. lost at $100^{\circ}$ C. 0.4964 grm.

II. 1.2599 gr. of substance dried at $100^{\circ} \mathrm{C}$. left, after incineration, $0.0084 \mathrm{gr}$.

Pectic acid, dried at $100^{\circ} \mathrm{C}$. -

Pectine combined with $\mathrm{PbO}$.

$$
\begin{aligned}
& \text { (Regnault) } \ldots \ldots \ldots \ldots \ldots \ldots, \mathrm{C}_{12} \\
& \text { (Mulder) } \ldots \ldots \ldots \ldots \ldots \ldots \mathrm{C}_{12}
\end{aligned} \mathrm{H}_{8} \mathrm{H}_{10^{\circ}} \mathrm{O}_{11}
$$

(Fremy) ............... $\mathrm{C}_{10} \mathrm{H}_{9} \mathrm{O}_{11}$.

Phil. Mag. S. 3. Vol. 29. No. 195. Nov. 1846. 
III. 0.3643 gr. gave 0.6015 gr. $\mathrm{CO}^{2}$ and $0.2175 \mathrm{gr}$. HO. IV. 0.5429 gr. gave $0.9022 \mathrm{gr}$. $\mathrm{CO}^{2}$ and $0.1350 \mathrm{gr}$. HO.

V. $0.8705 \mathrm{gr}$. gave, by the method of Varrentrapp and Will, 0.2974 gr. ammonio-chloride of platinum. num.

VI. 0.698 gr. gave 0.2331 gr. ammonio-chloride of plati-

Expressed in 100 parts, the above give-

\begin{tabular}{lrr} 
Carbon ... & \multicolumn{1}{c}{ I. } & \multicolumn{1}{c}{ II. } \\
Hydrogen . & $6 \cdot 67$ & $45 \cdot 32$ \\
Nitrogen ... & $2 \cdot 14$ & $6 \cdot 63$ \\
Ashes ...... & $0 \cdot 67$ & $2 \cdot 10$ \\
Water...... & $13 \cdot 65$ &
\end{tabular}

Estimated in 100 parts, according to the method described at page 368, the above determinations yield the following numbers :-

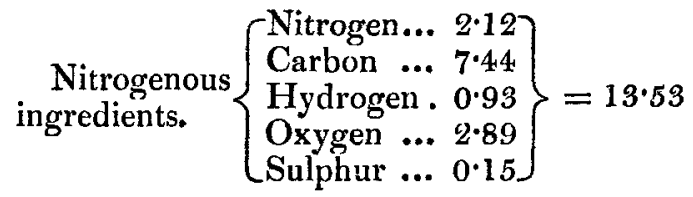

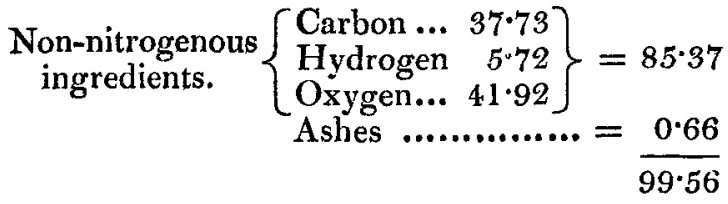

No. 3. Wheat-flour from Vienna.

I. $3.5345 \mathrm{grms}$. lost at $100^{\circ}$ C. $0.4500 \mathrm{gr}$.

II. $4.4785 \mathrm{grs}$. of substance dried at $100^{\circ} \mathrm{C}$. gave 0.04 .97 gr. ashes.

III. $0.5545 \mathrm{gr}$. gave $0.9339 \mathrm{gr} . \mathrm{CO}^{2}$ and $0.3377 \mathrm{gr}$. HO.

IV. 0.3310 gr. gave $0.5655 \mathrm{gr}$. $\mathrm{CO}^{2}$ and $0.2031 \mathrm{gr}$. HO.

V. $0.6405 \mathrm{gr}$. gave $0.3514 \mathrm{gr}$, of ammonio-chloride of platinum.

These determinations, expressed in 100 parts, give-

\begin{tabular}{lrr} 
& \multicolumn{1}{c}{ I. } & \multicolumn{1}{c}{ II. } \\
Carbon...... & 45.93 & 46.59 \\
Hydrogen & $6 \cdot 76$ & $6 \cdot 81$ \\
Nitrogen ... & $3 \cdot 44$ & \\
Ashes ...... & $1 \cdot 11$ & \\
Water ...... & $12 \cdot 73$ &
\end{tabular}

Estimated in 100 parts, as previously, the above determinations give the following numbers:- 


$$
\begin{aligned}
& \begin{array}{c}
\text { Nitrogenous } \\
\text { ingredients. }
\end{array} \quad\left\{\begin{array}{lr}
\text { Nitrogen... } & 3.44 \\
\text { Carbon ... } & 12.08 \\
\text { Hydrogen .. } & 1.50 \\
\text { Oxygen ... } & 4.68 \\
\text { Sulphur ... } & 0.25
\end{array}\right\}=21.93 \\
& \left.\begin{array}{l}
\text { Ingredients } \\
\text { containing no } \\
\text { nitrogen. }
\end{array} \begin{array}{lr}
\text { Carbon ... } & 34 \cdot 78 \\
\text { Hydrogen } & 5 \cdot 28 \\
\text { Oxygen ... } & 37 \cdot 97
\end{array}\right\}=78 \cdot 03 \\
& \text { Ashes................. }=1 \cdot 10 \\
& \overline{101 \cdot 06}
\end{aligned}
$$

Talavera Wheat (Triticum vulgare) from Hohenheim.

This variety is of high reputation as a winter grain. Berry yellow, of medium size, and slightly shrunk. 10 grains weigh 0.3606 grm.

I. $2 \cdot 3010 \mathrm{grs}$. lost at $100^{\circ} \mathrm{C} .0 .3551 \mathrm{gr}$.

II. 3.9868 grs. of substance dried at $100^{\circ}$ gave 0.1116 ashes. III. $0.2796 \mathrm{gr}$. gave $0.3915 \mathrm{gr}$. $\mathrm{CO}^{2}$ and $0.1672 \mathrm{gr}$. $\mathrm{HO}$. IV. $0.2387 \mathrm{gr}$, gave $0.3915 \mathrm{gr}$. $\mathrm{CO}^{2}$ and $0.1427 \mathrm{gr}$. $\mathrm{HO}$. V. 0.64 .29 gr. gave 0.2711 gr. ammonio-chloride of platinum.

These, expressed in parts of 100 , give-

I.

Carbon...... 45.14,

Hydrogen.. 6.64

Nitrogen .... 2.59

Ashes ...... 2.80

Water ....... $15 \cdot 43$
II.

$44 \cdot 73$

$5 \cdot 97$

Estimated in 100 parts, as previously, the above determinations give the following numbers:-

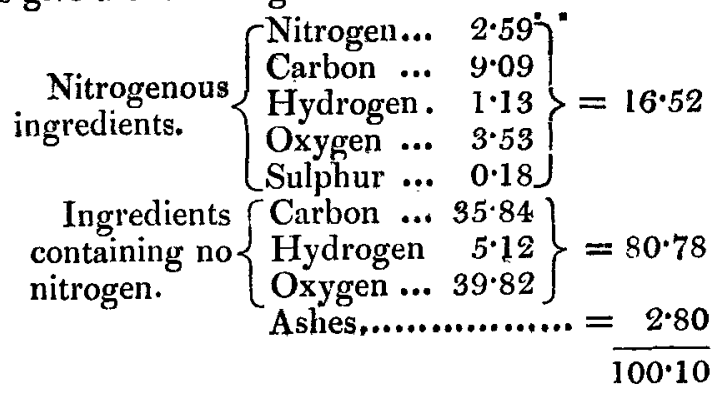

Whitington Wheat (Triticum vulgare) from Hohenheim.

An English variety of great excellence. Berry yellow, or white, large, and slightly shrunk. 10 grains weigh $0.4239 \mathrm{gr}$.

I. $2 \cdot 6221$ grs. lost at $100^{\circ}$ C. $0.3653 \mathrm{gr}$.

$$
2 \mathrm{C} 2
$$


II. $4.6567 \mathrm{grs}$. of substance dried at $100^{\circ} \mathrm{C}$. gave $0 \cdot 1460$ gr. ashes.

III. $0.2927 \mathrm{gr}$. gave $0.4747 \mathrm{gr}$. $\mathrm{CO}^{2}$ and $0.1834 \mathrm{gr}$. HO.

IV. $0.3898 \mathrm{gr}$. gave $0.6377 \mathrm{gr}$. $\mathrm{CO}^{2}$ and $0.2343 \mathrm{gr}^{\circ} \mathrm{HO}$. num.

V. 0.5494 gr. gave 0.2343 gr. ammonio-chloride of plati-

Expressed in 100 parts-

I.

Carbon...... 44.23

Hydrogen. . 6.96

Nitrogen ... $2 \cdot 68$

Ashes ...... 3.13

Water ...... 13.93
II.

$44 \cdot 61$

6.67

Estimaterl in 100 parts, as previously, the above determinations give the following numbers:-

$$
\begin{aligned}
& \text { Nitrogenous }\left\{\begin{array}{ll}
\text { Nitrogen... } & 2 \cdot 68 \\
\text { Carbon ... } & 9 \cdot 40 \\
\text { Hydrogen } & 1 \cdot 17 \\
\text { Oxygen ... } & 3 \cdot 65 \\
\text { Sulphur ... } & 0 \cdot 19
\end{array}\right\}=17 \cdot 09 \\
& \text { Ingredients Carbon ... 35.02 } \\
& \text { containing no }\left\{\begin{array}{lr}
\text { Hydrogen } & 5.65 \\
\text { Oxygen } & 38.91
\end{array}=79.58\right. \\
& \text { nitrogen. } \\
& \text { Oxygen ... 38.91 } \\
& \text { Ashes.................. }=\frac{3 \cdot 18}{99 \cdot 80}
\end{aligned}
$$

Sandomierz Wheat (Triticum vulgare) from Hohenheim.

Berry scarcely of medium size, plump and sound. This variety is known as one of the best in Germany. 10 grains weigh $0.3199 \mathrm{grm}$.

I. $4.2256 \mathrm{grs}$. lost at $100^{\circ} \mathrm{C} .0 .6545 \mathrm{gr}$. ashes.

II. $3.6555 \mathrm{grs}$. of substance dried at $100^{\circ}$ gave $0.0886 \mathrm{gr}$.

III. 0.5754 gr. gave $0.9327 \mathrm{gr} . \mathrm{CO}^{2}$ and $0.3465 \mathrm{gr}$. HO.

IV. 0.7303 gr. gave $0.3131 \mathrm{gr}$. ammonio-chloride of platinum.

Expressed in 100 parts-

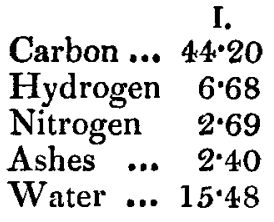

Estimated in 100 parts, as previously, the above determinations give the following numbers:- 


$$
\begin{aligned}
& \text { Nitrogenous }\left\{\begin{array}{lll}
\text { Nitrogen... } & 2 \cdot 69 \\
\text { Carbon ... } & 9 \cdot 44 \\
\text { Hydrogen.. } & 1 \cdot 17 \\
\text { Oxygen ... } & 3.66 \\
\text { Sulphur ... } & 0 \cdot 19
\end{array}\right\}=17 \cdot 15 \\
& \begin{array}{l}
\text { Ingredients } \\
\text { containing no } \\
\text { nitrogen. }
\end{array}\left\{\begin{array}{lr}
\text { Carbon ... } & 34 \cdot 76 \\
\text { Hydrogen. } & 5 \cdot 51 \\
\text { Oxygen ... } & 38 \cdot 62
\end{array}\right\}=78 \cdot 89 \\
& \text { Ashes................. }=\frac{2 \cdot 40}{98 \cdot 44}
\end{aligned}
$$

No. 1. Rye-flour from Vienna.

I. $3.253 \mathrm{grs}$. at $100^{\circ}$ lost $0.4482 \mathrm{gr}$.

II. $2 \cdot 889$ grs. of the substance dried at $100^{\circ}$ gave 0.0387 gr. ashes.

III. 3.2285 grs. gave 0.0427 ashes.

IV. 0.3383 gr. gave $0.5576 \mathrm{gr}$. $\mathrm{CO}^{2}$ and $0.1983 \mathrm{gr}$. HO.

V. $0.3109 \mathrm{gr}$. gave $0.5055 \mathrm{gr} . \mathrm{CO}^{2}$ and $0.1901 \mathrm{gr}$. HO.

VI. $0.6519 \mathrm{gr}$. gave $0 \cdot 194 \mathrm{l} \mathrm{gr}$. ammonio-chloricle of platinum.

These correspond in 100 parts to-

$$
\begin{array}{lrr} 
& \text { I. } & \multicolumn{1}{c}{\text { II. }} \\
\text { Carbon...... } & 44 \cdot 41 & 44 \cdot 34 \\
\text { Hydrogen.. } & 6 \cdot 51 & 6.79 \\
\text { Nitrogen ... } & 1 \cdot 87 & \\
\text { Ashes ...... } & 1 \cdot 34 & 1 \cdot 32 \\
\text { Water...... } & 13.78 &
\end{array}
$$

Estimated in 100 parts, as previously, the above determinations give the following numbers:-

$$
\begin{aligned}
& \text { Nitrogenous }\left\{\begin{array}{lll}
\text { Nitrogen... } & 1 \cdot 87 \\
\text { Carbon ... } & 6 \cdot 56 \\
\text { Hydrogen.. } & 0 \cdot 82 \\
\text { Oxygredients. } & . . & 2 \cdot 54 \\
\text { Sulphur ... } & 0 \cdot 13
\end{array}\right\}=11.92
\end{aligned}
$$

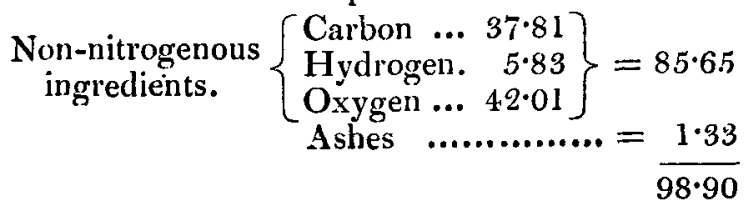

No. 2. Rye-flour from Vienna.

I. $7 \cdot 4.625$ grs. lost at $100^{\circ}$ C. $1.0956 \mathrm{gr}$.

II. $2 \cdot 8000 \mathrm{grs}$. of substance dried at $100^{\circ} \mathrm{C}$. gave $0 \cdot 0300$ gr. ashes.

III. $0.5312 \mathrm{gr}$. gave $0.8752 \mathrm{gr}$. $\mathrm{CO}^{2}$ and $0.3116 \mathrm{gr}$. HO. 
IV. $0.4577 \mathrm{gr}$. gave $0.7626 \mathrm{gr}$. $\mathrm{CO}^{2}$ and $0.2720 \mathrm{gr}$. HO.

V. 0.7558 gr. gave 0.3537 gr. atnmonio-chloride of platinum.

VI. 0.7378 gr. gave 0.3433 gr. ammonio-chloride of platinum.

These correspond in 100 parts to-

$\begin{array}{lrr} & \text { I. } & \text { II. } \\ \text { Carbon...... } & 4.4 \cdot 94 & 45 \cdot 44 \\ \text { Hydrogen.. } & 6 \cdot 52 & 6 \cdot 60 \\ \text { Nitrogen ... } & 2 \cdot 94 & 2 \cdot 92 \\ \text { Ashes ...... } & 1 \cdot 07 & \\ \text { Water ...... } & 14 \cdot 68 & \end{array}$

Estimated in 100 parts, as previously, the above determinations give the following numbers:-

$$
\begin{aligned}
& \begin{array}{c}
\text { Nitrogenous } \\
\text { ingredients. }
\end{array}\left\{\begin{array}{lr}
\text { Nitrogen... } & 2.93 \\
\text { Carbon ... } & 10.28 \\
\text { Hydrogen. } & 1.28 \\
\text { Oxygen ... } & 3.99 \\
\text { Sulphur ... } & 0.21
\end{array}\right\}=18.69
\end{aligned}
$$

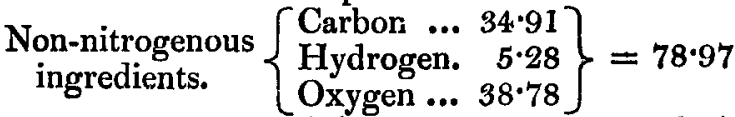

$$
\begin{aligned}
& \text { Ashes................. }=1.07 \\
& \overline{98 \cdot 73}
\end{aligned}
$$

Bush-rye (Secale cereale) from Hohenheim.

Winter crop. In the Hohenheim catalogue is the following remark :- " Besides its other qualities, it yields such excellent straw that it deserves being mentioned." The berry is small and generally shrunken. 10 grains weigh $0.1220 \mathrm{grm}$.

I. 4.2303 grms. lost at $100^{\circ}$ C. $0.5896 \mathrm{gr}$.

II. 4.3792 grs. of substance dried at $100^{\circ} \mathrm{C}$. gave 0.1078 gr. ashes.

III. $0.4742 \mathrm{gr}$. gave $0 \cdot 7879 \mathrm{gr} . \mathrm{CO}^{2}$ and $0.2767 \mathrm{gr}$. HO.

IV. 0.6281 gr. gave $1.0555 \mathrm{gr}$. $\mathrm{CO}^{2}$ and $0.3777 \mathrm{gr}$. $\mathrm{HO}$.

V. 0.6807 gr. gave 0.3016 gr. ammonio-chloride of platinum.

These correspond in 100 parts to-

$$
\begin{array}{lrr} 
& \text { 1. } & \text { II. } \\
\text { Carbon...... } & 45 \cdot 31 & 45 \cdot 83 \\
\text { Hydrogen. } & 6 \cdot 48 & 6 \cdot 68 \\
\text { Nitrogen ... } & 2 \cdot 78 & \\
\text { Ashes ...... } & 2 \cdot 43 & \\
\text { Water ...... } & \mathbf{1 3} \cdot 94 & \\
\hline
\end{array}
$$

Estimated in 100 parts, as previously, the above determinations give the following numbers:- 


$$
\begin{aligned}
& \text { Nitrogenous }\left\{\begin{array}{lll}
\text { Nitrogen ... } & 2 \cdot 78 \\
\text { Carbon ... } & 9 \cdot 76 \\
\text { ingredients. } & \text { Hydrogen. } & 1 \cdot 21 \\
\text { Oxygen ... } & 3 \cdot 78 \\
\text { Sulphur ... } & 0 \cdot 20
\end{array}\right\}=17 \cdot 73 \\
& \begin{array}{c}
\text { Non-nitrogenous } \\
\text { ingredients. }
\end{array}\left\{\begin{array}{lr}
\text { Carbon ... } & 35 \cdot 76 \\
\text { Hydrogen. } & 5 \cdot 37 \\
\text { Oxygen ... } & 39 \cdot 73
\end{array}\right\}=80 \cdot 86 \\
& \text { Ashes................ }=2 \cdot 43 \\
& \overline{101.02}
\end{aligned}
$$

Rush-rye (Secale cereale arundinaceum) from Hohenheim. Berry of medium size and slightly shrunken. 10 grains weigh $0 \cdot 1838 \mathrm{grm}$.

I. 3.3139 grms. lost at $100^{\circ} \mathrm{C} .0 .4579 \mathrm{gr}$.

II. $1.4596 \mathrm{gr}$. of substance dried at $100^{\circ}$ gave $0.0346 \mathrm{gr}$. ashes.

III. $0.2795 \mathrm{gr}$. gave $0.4620 \mathrm{gr}$. $\mathrm{CO}^{2}$ and $0.1742 \mathrm{gr}$. HO.

IV. 0.2632 gr. gave $0.4381 \mathrm{gr}$. $\mathrm{CO}^{2}$ and $0.1638 \mathrm{gr}$. HO.

V. 0.6435 gr. gave 0.2530 gr. ammonio-chloride of platinum.

These, expressed in 100 parts, give-

$$
\begin{array}{lrr} 
& \multicolumn{1}{c}{\text { I. }} & \multicolumn{1}{c}{\text { II. }} \\
\text { Carbon...... } & 45 \cdot 08 & \mathbf{4 5 \cdot 3 9} \\
\text { Hydrogen.. } & 6 \cdot 92 & 6 \cdot 22 \\
\text { Nitrogen ... } & 2 \cdot 47 & \\
\text { Ashes ...... } & 2 \cdot 37 & \\
\text { Water ...... } & 13.82 &
\end{array}
$$

Estimated in 100 parts, the above determinations give the following numbers:-

$$
\begin{aligned}
& \text { Nitrogenous }\left\{\begin{array}{lll}
\text { Nitrogen... } & 2 \cdot 47 \\
\text { Carbon ... } & 8 \cdot 67 \\
\text { Hydrogen. } & 1 \cdot 08 \\
\text { Oxygen ... } & 3 \cdot 36 \\
\text { Sulphur ... } & 0 \cdot 18
\end{array}\right\}=15 \cdot 76 \\
& \text { Non-nitrogenous } \text { Carbon ... 36.56 } \\
& \text { ingredients. }\{\text { Hydrogen. } 5.49\}=82.67 \\
& \text { Oxygen ... 40.62 } \\
& \text { Ashes................. }=2.37 \\
& \overline{100 \cdot 80}
\end{aligned}
$$

Polenta Meal (Indian corn-meal) from Vienna.

Yellow and coarse.

I. $5.5810 \mathrm{grs}$. lost at $100^{\circ} 0.7456 \mathrm{gr}$.

II. 4.3933 grs. of substance dried at $100^{\circ}$ C. gave 0.0386 gr. ashes. 
III. $3.9323 \mathrm{gr}$. gave $0.331 \mathrm{gr}$, ashes.

IV. $0.5444 \mathrm{gr}$. gave $0.9010 \mathrm{gr}$. $\mathrm{CO}^{2}$ and $0.3330 \mathrm{gr}$. HO.

V. 0.5095 gr. gave 0.8399 gr. $\mathrm{CO}^{2}$ and 0.2943 gr. HO. num.

VI. 0.9055 gr. gave 0.3093 gr. ammonio-chloride of plati-

These correspond in 100 parts to-

$$
\begin{array}{lrrr} 
& \multicolumn{1}{c}{\text { I. }} & \multicolumn{1}{c}{\text { II. }} \\
\text { Carbon...... } & 45 \cdot 14 & 44 \cdot 96 \\
\text { Hydrogen... } & 6 \cdot 89 & 6.49 \\
\text { Nitrogen ... } & 2 \cdot 14 & \\
\text { Ashes ...... } & 0.87 & 0.84 \\
\text { Water ...... } & 13.36 &
\end{array}
$$

Estimated in 100 parts, the above determinations give the following numbers:-

$$
\begin{aligned}
& \text { Nitrogenous }\left\{\begin{array}{lll}
\text { Nitrogen... } & 2 \cdot 14 \\
\text { Carbon ... } & 7 \cdot 51 \\
\text { Hydrogen. } & 0 \cdot 93 \\
\text { ingredients. } & \text { Oxygen ... } & 2 \cdot 92 \\
\text { Sulphur ... } & 0 \cdot 15
\end{array}\right\}=13 \cdot 65
\end{aligned}
$$

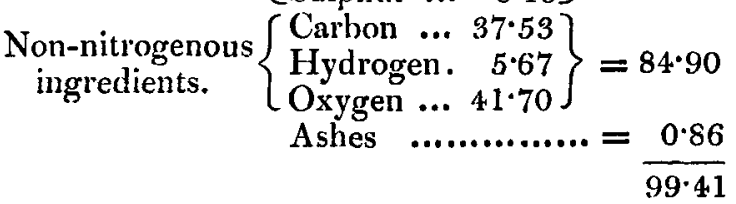

Common Yellow Maize, Indian corn (Zea mays), from Hohenheim.

Yellow oval berry, bright and sound. 10 grains weigh 3.5934 grms.

I. 4.5765 grs. lost at $100^{\circ}$ C. $0.6849 \mathrm{gr}$.

II. 5.0654 gr's. of substance dried at $100^{\circ} \mathrm{C}$. gave 0.0974 gr. ashes.

III. $0.4164 \mathrm{gr}$, gave $0.6984 \mathrm{gr}$. $\mathrm{CO}^{2}$ and $0.2468 \mathrm{gr}$. $\mathrm{HO}$.

IV. $0.4103 \mathrm{gr}$. gave $0.6800 \mathrm{gr}$. $\mathrm{CO}^{2}$ and $0.2454 \mathrm{gr}$. HO.

V. 0.7319 gr. gave $0.2684 \mathrm{gr}$. ammonio-chloride of platinum.

These correspond in 100 parts to-

$$
\begin{array}{lrr} 
& \text { I. } & \multicolumn{1}{c}{\text { II. }} \\
\text { Carbon...... } & 4 \cdot 5 \cdot 74 & 4.5 \cdot 20 \\
\text { Hydrogen.. } & 6 \cdot 58 & 6 \cdot 64 \\
\text { Nitrogen ... } & \mathbf{2 \cdot 3 0} & \\
\text { Ashes ...... } & 1 \cdot 92 & \\
\text { Water ...... } & 14 \cdot 96 &
\end{array}
$$

Estimated in 100 parts, the above determinations give the following numbers:- 


$$
\begin{aligned}
& \text { Nitrogenous }\left\{\begin{array}{lll}
\text { Nitrogen... } & 2 \cdot 30 \\
\text { Carbon ... } & 8 \cdot 07 \\
\text { Hydrogen.: } & 1 \cdot 00 \\
\text { Oxygen ... } & 3 \cdot 13 \\
\text { Sulphur ... } & 0 \cdot 16
\end{array}\right\}=14 \cdot 66
\end{aligned}
$$

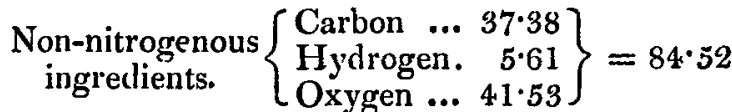

$$
\begin{aligned}
& \text { Ashes................ }=1.92 \\
& \overline{101 \cdot 10}
\end{aligned}
$$

Triticum monococcum from Hohenheim.

I. 4.4914 grs. lost at $100^{\circ} \mathrm{C} .0 .6472 \mathrm{gr}$.

II. $7 \cdot 1927$ grs. of substance dried at $100^{\circ} \mathrm{C}$. gave $0 \cdot 1438$ gi. ashes.

III. $0.6303 \mathrm{gr}$. gave $1.0288 \mathrm{gr}$. $\mathrm{CO}^{2}$ and $0.38 \mathrm{ll} \mathrm{gr}$. HO.

IV. $0.6757 \mathrm{gr}$. gave $1 \cdot 104 \mathrm{gr}$ gr. $\mathrm{CO}^{2}$ and $0.4098 \mathrm{gr}$. HO.

V. 0.7105 gr. gave 0.2340 gr. ammonio-chloride of platinum.

These correspond in 100 parts to-

\section{I.}

Carbon...... 44:51

Hydrogen. . 6.71

Nitrogen ... 2.07

Ashes ...... 2.01

Water ...... 14:40
II.

$44 \cdot 56$

$6 \cdot 74$

Estimated in 100 parts, the above determinations give the following numbers :-

$$
\begin{aligned}
& \text { Nitrogenous }\left\{\begin{array}{lll}
\text { Nitrogen ... } & 2 \cdot 07 \\
\text { Carbon ... } & 7 \cdot 26 \\
\text { Hydrogen. } & 0 \cdot 90 \\
\text { Oxygen ... } & 2 \cdot 82 \\
\text { Sulphur ... } & 0 \cdot 15
\end{array}\right\}=13 \cdot 20
\end{aligned}
$$

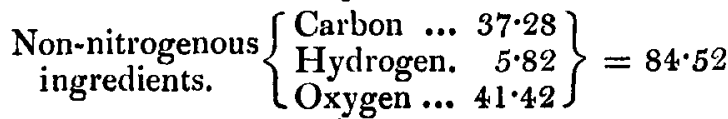

$$
\begin{aligned}
& \text { Ashes } \ldots . . . \ldots \ldots \ldots=\frac{2.01}{99.73}
\end{aligned}
$$

Jerusalem Barley (Hordeum distichon) from Hohenheim.

10 grains weigh $0.5312 \mathrm{grm}$.

I. $1.9328 \mathrm{grm}$. lost at $100^{\circ}$ C. $0.3247 \mathrm{grm}$. 
II. $2.3553 \mathrm{grs}$, of substance dried at $100^{\circ} \mathrm{C}$, gave 0.0670 gr. ashes.

III. $0.4457 \mathrm{gr}$. gave $0.7392 \mathrm{gr} . \mathrm{CO}^{2}$ and $0.2787 \mathrm{gr}$. HO.

IV. $0.4603 \mathrm{gr}$. gave $0 \cdot 7728 \mathrm{gr}$. $\mathrm{CO}^{2}$ and $0.2913 \mathrm{gr}$. HO. num.

V. $0.6910 \mathrm{gr}$. gave $0.2560 \mathrm{gr}$. ammonio-chloride of plati-

These determinations correspond in 100 parts to-

I.

Carbon...... 45.23

Hydrogen... 6.94

Nitrogen ... $2 \cdot 31$

Ashes ...... 2.84

Water ...... $16 \cdot 79$
II.

$45 \cdot 78$

$6 \cdot 79$

Estimated in 100 parts, the above determinations give the following numbers:-

$$
\begin{aligned}
& \text { Nitrogenous }\left\{\begin{array}{lll}
\text { Nitrogen ... } & 2 \cdot 34 \\
\text { Carbon ... } & 8 \cdot 11 \\
\text { Hydrogredients. } & \text { Hydrogen. } & 1 \cdot 00 \\
\text { Oxygen ... } & 3 \cdot 14 \\
\text { Sulphur ... } & 0 \cdot 16
\end{array}\right\}=14 \cdot 72
\end{aligned}
$$

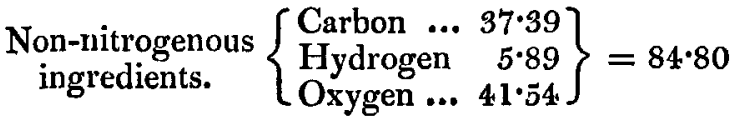

$$
\begin{aligned}
& \text { Ashes................. }=\frac{2 \cdot 84}{102 \cdot 36}
\end{aligned}
$$

Common Winter Barley (Hordeum vulgare) from Hohenheim.

10 grains weigh 0.3955 grm.

I. $1.5268 \mathrm{gr}$. lost at $100^{\circ}$ C. $0.2107 \mathrm{gr}$.

II. $2 \cdot 5708$ grs. of substance dried at $100^{\circ}$ C. gave $0 \cdot 1409$ gr. ashes.

III. $0.3244 \mathrm{gr}$, gave $0.5380 \mathrm{gr}$. $\mathrm{CO}^{2}$ and $0.1928 \mathrm{gr}$. $\mathrm{HO}$.

IV. $0.2505 \mathrm{gr}$. gave $0.4152 \mathrm{gr}$. $\mathrm{CO}^{2}$ and $0.1611 \mathrm{gr}$. HO. num.

V. 0.5266 gr. gave $0.2342 \mathrm{gr}$. ammonio-chloride of plati-

VI. 4.3619 grs. of grains (calculated upon substance dried at $100^{\circ} \mathrm{C}$.) gave, by the method already described, page 368, $0.2356 \mathrm{gr}$. woody fibre and combined inorganic matter.

VII. $0.1793 \mathrm{gr}$. of the same woodty fibre gave $0.0035 \mathrm{gr}$. ashes.

These, expressed in 100 parts, correspond to-

\begin{tabular}{lrr} 
& \multicolumn{1}{c}{ I. } & \multicolumn{1}{c}{ II. } \\
Carbon...... & $45 \cdot 23$ & $45 \cdot 20$ \\
Hydrogen ... & $6 \cdot 60$ & $7 \cdot 14$ \\
Nitrogen ... & $\mathbf{2 \cdot 7 9}$ &
\end{tabular}


of different kinds of Vegetable Food.

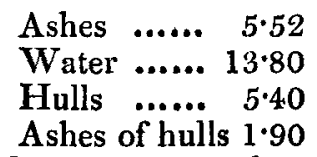

Estimated in 100 parts, the above determinations give the following numbers :-

$$
\begin{aligned}
& \text { Nitrogenous }\left\{\begin{array}{lll}
\text { Nitrogen .. } & 2 \cdot 79 \\
\text { Carbon ... } & 9 \cdot 79 \\
\text { Hydrogen } & 1 \cdot 12 \\
\text { Oxygen ... } & 3 \cdot 80 \\
\text { Sulphur ... } & 0 \cdot 20
\end{array}\right\}=17 \cdot 70
\end{aligned}
$$

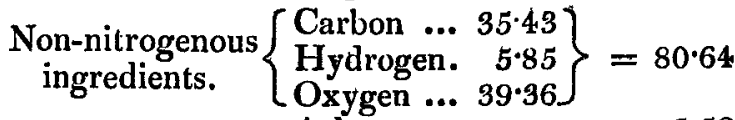

$$
\begin{aligned}
& \text { Ashes.................. }=\frac{5.52}{103.86} \\
& \overline{100 \cdot 00} \\
& 100 \cdot 00
\end{aligned}
$$

Kamskatka Oats (Avena sativa) from Hohenheim.

10 grains weigh $0.3446 \mathrm{gr}$.

I. $2.3657 \mathrm{grs}$. Iost at $100^{\circ}$ C. $0.3446 \mathrm{gr}$.

II. $3 \cdot 1728 \mathrm{gr}$. of substance dried at $100^{\circ} \mathrm{C}$. gave 0.1032 gr, ashes.

III. $0.4 .310 \mathrm{gr}$. gave $0.734 \mathrm{I}$ gr. $\mathrm{CO}^{2}$ and $0.2558 \mathrm{gr}$. HO.

IV. $0.4001 \mathrm{gr}$. gave $0.6830 \mathrm{gr} . \mathrm{CO}^{2}$ and $0.2427 \mathrm{gr}$. HO.

V. $0.4102 \mathrm{gr}$. gave $0.1581 \mathrm{gr}$. ammonio-chloride of platinum.

VI. $0.6207 \mathrm{gr}$. of the same gave $0.2324 \mathrm{gr}$. ammonio-chloride of platinum.

These, expressed in 100 parts, correspond to-

\begin{tabular}{lrr} 
& \multicolumn{1}{c}{ I. } & \multicolumn{1}{c}{ II. } \\
Carbon ... & $46 \cdot 45$ & $46 \cdot 55$ \\
Hydrogen & $6 \cdot 55$ & $6 \cdot 73$ \\
Nitrogen... & $2 \cdot 42$ & $2 \cdot 35$ \\
Ashes...... & $3 \cdot 26$ & \\
Water ... & $12 \cdot 71$ &
\end{tabular}

Estimated in 100 parts, the above determinations give the following numbers :- 


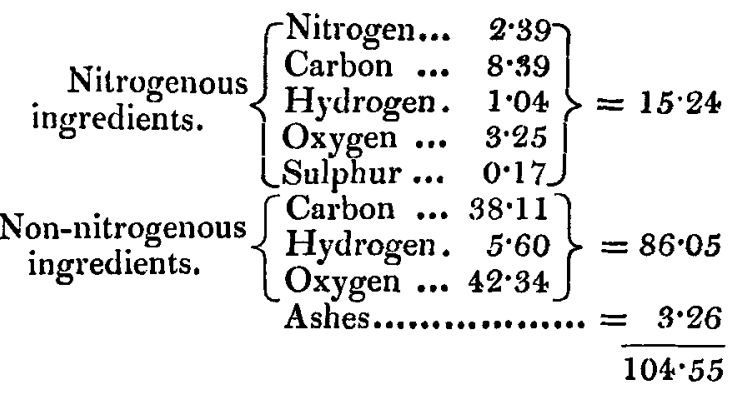

Early White-panicled Oats (Avena sativa) from Hohenheim.

Berry bright, plump and sound. 10 grains weigh $0.3689 \mathrm{gr}$. I. $1 \cdot 7548 \mathrm{gr}$. lost at $100^{\circ}$ C. $02271 \mathrm{gr}$.

II. $1.8486 \mathrm{gr}$. of substance dried at $100^{\circ} \mathrm{C}$. gave $0.0765 \mathrm{gr}$. ashes.

III. $0.3539 \mathrm{gr}$. gave $0.6057 \mathrm{gr}$. $\mathrm{CO}^{2}$ and $0.2144 \mathrm{gr}$. HO.

IV. $0.2410 \mathrm{gr}$. gave $0.4123 \mathrm{gr}$. $\mathrm{CO}^{2}$ and $0.1451 \mathrm{gr}$. HO.

V. $0.4977 \mathrm{gr}$. gave $0.2236 \mathrm{gr}$. ammonio-chloride of platinum.

VI. 3.5498 grs. gave, by the method already described, $0.5916 \mathrm{gr}$. woody fibre and combined inorganic matter.

VII. $0.4197 \mathrm{gr}$. of the same woody fibre gave $0.0140 \mathrm{gr}$. ashes.

Expressed in 100 parts, these correspond with-

1 .

Carbon ...... 46.68

Hydrogen ... 6.73

Nitrogen ...... 2.82

Ashes ......... 4.14

Water......... 12.94

Hulls ......... 16.66

Ashes of hulls 3.35
II.

$46 \cdot 66$

$6 \cdot 69$

Estimated in 100 parts, the above determinations give the following numbers:-

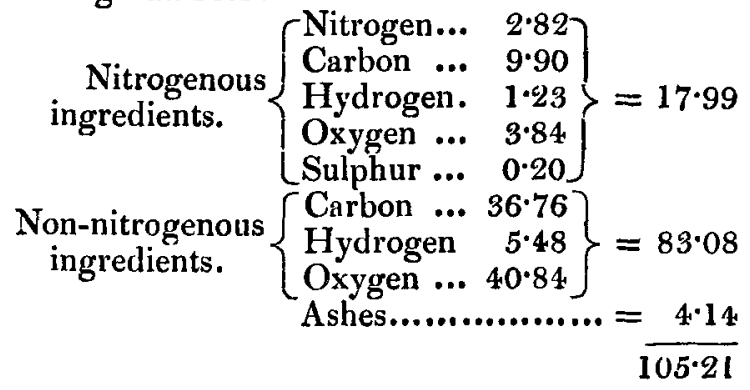




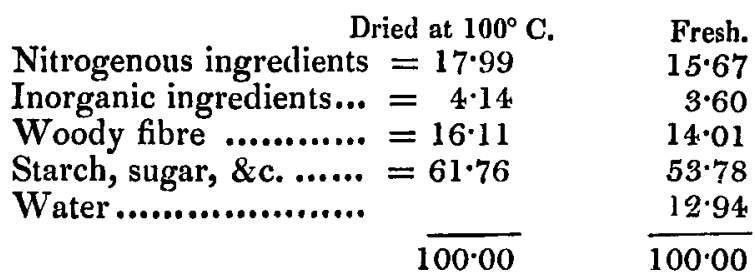

As the woody fibre, $=16^{\prime} 66$ per cent. of the whole, belongs mostly to the chaff, this grain ranks among the richest in nitrogenous compounds. 2.82 per cent. of nitrogen with the chaff equals 3.38 per cent. without.

Common Rice (Oryza sativa).

I. $7 \cdot 4606$ grs. lost at $100^{\circ}$ C. $1 \cdot 1301 \mathrm{gr}$.

II. $8.3670 \mathrm{grs}$ of substance dried at $100^{\circ} \mathrm{C}$. gave 0.0306 gr. ashes.

III. $0.7158 \mathrm{gr}$. gave $1.1701 \mathrm{gr}$. $\mathrm{CO}^{2}$ and $0.4244 \mathrm{gr}$. $\mathrm{HO}$.

IV. $0.6095 \mathrm{gr}$ gave $0.9982 \mathrm{gr} . \mathrm{CO}^{2}$ and $0.3559 \mathrm{gr}$. $\mathrm{HO}$.

V. 1.5609 gr. gave 0.2892 gr. ammonio-chloride of platinum.

These determinations correspond in 100 parts to-

I.

Carbon ...4 44.57

Hydrogen 6.58

Nitrogen... $1 \cdot 16$

Ashes...... 0:36

Water ... $15 \cdot 14$
II.

$4 \cdot 4 \cdot 66$

$6 \cdot 48$

Estimated in 100 parts, these determinations give the following numbers :-

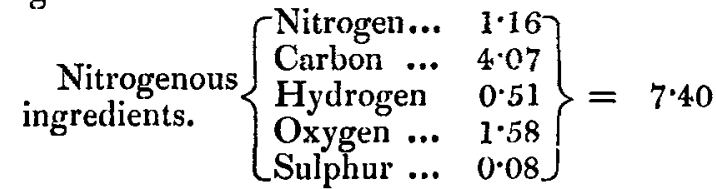

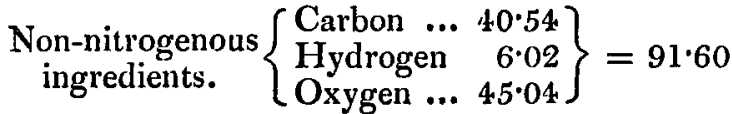

$$
\begin{aligned}
& \text { Ashes } \ldots . . . . . . . . .=\frac{0.36}{99.36}
\end{aligned}
$$

Buckwheat-meal from Vienna.

I. 7.3962 grs. lost at $100^{\circ} \mathrm{C} .1 \cdot 1187 \mathrm{gr}$.

II. $5 \cdot 1500 \mathrm{grs}$. of substance dried at $100^{\circ} \mathrm{C}$. gave 0.0564 gr. ashes.

III. $0.5041 \mathrm{gr}$, gave $0.8194 \mathrm{gr}, \mathrm{CO}^{2}$ and $0.2911 \mathrm{gr}$. HO. 
IV. $0.344 .1 \mathrm{gr}$. gave $0.5577 \mathrm{gr} . \mathrm{CO}^{2}$ and $0.2061 \mathrm{gr}$. HO.

V. $1 \cdot 1295$ gr, gave $0.2062 \mathrm{gr}$, ammonio-chloride of platinum. num.

VI. 0.9536 gr. gave 0.1561 gr. ammonio-chloride of plati-

These, expressed in 100 parts, correspond to-

\begin{tabular}{lcr} 
& I. & \multicolumn{1}{c}{ II. } \\
Carbon ... & 44.36 & 44.20 \\
Hydrogen & $6 \cdot 4.2$ & 6.68 \\
Nitrogen.. & $1 \cdot 14$ & 1.03 \\
Ashes.... & $1 \cdot 09$ & \\
Water .... & $15 \cdot 12$ &
\end{tabular}

Estimated in 100 parts, the above determinations give the following numbers :-

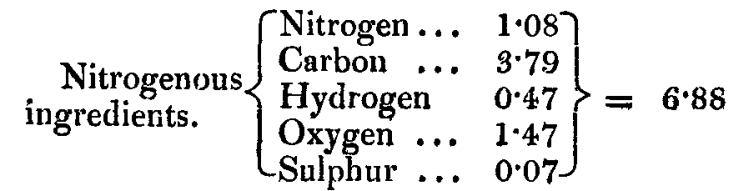

$$
\begin{aligned}
& \begin{array}{c}
\text { Non-nitrogenous } \\
\text { ingredients. }
\end{array}\left\{\begin{array}{lr}
\text { Carbon ... } & 40 \cdot 48 \\
\text { Hydrogen } & 6.07 \\
\text { Oxygen ... } & 44.97
\end{array}\right\}=91.52 \\
& \text { Ashes. . . . . . . . . }=\frac{1.09}{99.49}
\end{aligned}
$$

Tartarian Buckwheat (Polygonum tartaricum) from Hohenheim.

This variety differs from the common species, Polygonum Fagopyrum, in that while the hulls of the latter are smooth, those of the former are covered with folds and excrescences. The whole grain, hull included, was pnlverized and analysed. 10 grains weigh $0.2566 \mathrm{grm}$.

I. 2.7598 grs. lost at $100^{\circ}$ C. $0.3916 \mathrm{gr}$.

II. 2.5924 grs. of substance dried at $100^{\circ} \mathrm{C}$. gave 0.0597 gi. ashes.

III. 0.424 .5 gr. gave $0.7013 \mathrm{gr}$. $\mathrm{CO}^{2}$ and $0.2503 \mathrm{gr}$. HO.

IV. $0.3401 \mathrm{gr}$. gave $0.5710 \mathrm{gr} . \mathrm{CO}^{2}$ and $0.194 .3 \mathrm{gr}$. HO.

V. $0.5677 \mathrm{gr}$. gave $0.1407 \mathrm{gr}$. ammonio-chloride of platinum.

VI. 5.0444 grs. of the same, by the method already described, gave $1 \cdot 1438 \mathrm{gr}$. woody fibre and combined inorganic matter.

VII. $0.7100 \mathrm{gr}$. of the woody-fibre gave $0.0006 \mathrm{gr}$. ashes.

These, expressed in 100 parts, correspond to- 
of different kinds of Vegetable Food.

383

I.

Carbon $\quad \ldots 4.5 \cdot 06$

Hydrogen... 6.55

Nitrogen ... 1.56

Ashes ...... 2.30

Water ..... 14.19

Hulls ...... 22.67

Ashes of hulls 0.08
II.

$45 \cdot 79$

$6 \cdot 35$

Estimated in 100 parts, the above determinations give the following numbers :-

$$
\begin{aligned}
& \text { Nitrogenous }\left\{\begin{array}{lll}
\text { Nitrogen ... } & 1.56 \\
\text { Carbon ... } & 5 \cdot 47 \\
\text { Hydrogen } & 0.68 \\
\text { Oxygen } \ldots . . & 2.12 \\
\text { Sulphur ... } & 0.11
\end{array}\right\}=9 \cdot 94
\end{aligned}
$$

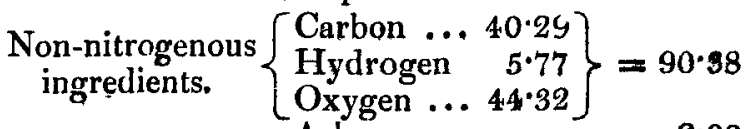

$$
\begin{aligned}
& \text { Ashes .......... } 2 \cdot 30 \\
& \overrightarrow{102 \cdot 62}
\end{aligned}
$$

Table Peas (Pisum sativum) from Vienna.

Bright, plump, sound and of medium size. 10 peas weigh $2 \cdot 6080$ grms.

I. $4 \cdot 4804$ grs. lost at $100^{\circ}$ C. $0.6018 \mathrm{gr}$.

II. $2 \cdot 1600$ grs. substance dried at $100^{\circ}$ C. gave $0 \cdot 0687$ gr. o ashes.

III. $0.4527 \mathrm{gr}$. gave $0.7476 \mathrm{gr}$. $\mathrm{CO}^{2}$ and $0.2789 \mathrm{gr}$. HO.

IV. $0.3810 \mathrm{gr}$. gave $0.6314 \mathrm{gr} . \mathrm{CO}^{2}$ and $0.2272 \mathrm{gr}$. $\mathrm{HO}$.

V. 0.8603 gr. gave 0.6047 gr. ammonio-ehloride of platinum.

VI. $5 \cdot 2332$ grs. of the same, according to the method described, gave $0 \cdot 4005 \mathrm{gr}$. hulls.

VII. $0.3962 \mathrm{gr}$. of hulls dried at $100^{\circ} \mathrm{C}$. gave $0.0098 \mathrm{gr}$. ashes.

These correspond in 100 parts to-

\begin{tabular}{lr} 
Carbon ... & \multicolumn{1}{c}{ I. } \\
Hydrogen & $6 \cdot 84$ \\
Nitrogen & $4 \cdot 42$ \\
Ashes .... & $3 \cdot 18$ \\
Water .... & $13 \cdot 4 \cdot 3$ \\
Hulls ..... & $7 \cdot 65$ \\
Ashes of hulls $2 \cdot 47$
\end{tabular}

II.

$45 \cdot 20$

6.62 
Estimated in 100 parts, the above determinations give the following numbers:-

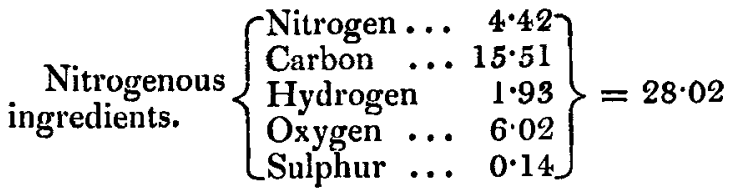

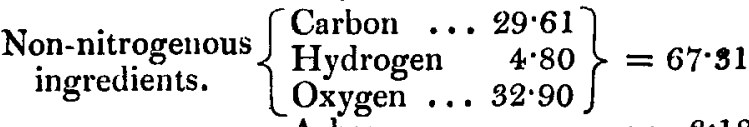

$$
\begin{aligned}
& \text { Ashes ............ }=\frac{3.18}{98.51}
\end{aligned}
$$

Legumine, according to the analysis of Dumas and Cahours, gave for the above determinations $102.00 *$.

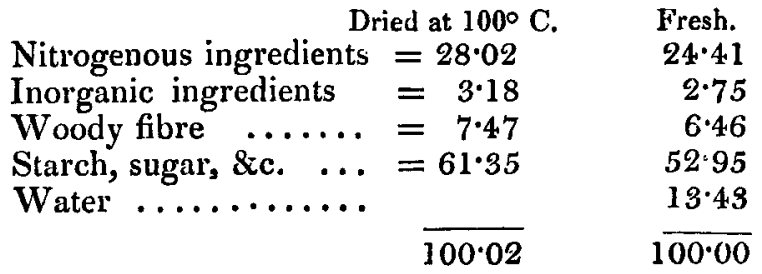

Field Peas (Pisum sativum) from Giessen.

Less in size than the preceding variety. 10 grains weigh $1.9829 \mathrm{grm}$.

I. 3.5904 grs. lost at $100^{\circ} 0.7002 \mathrm{gr}$.

II. $2 \cdot 2455$ grms. of substance dried at $100^{\circ} \mathrm{C}$. gave 0.0628 gr. ashes.

III. $0.6178 \mathrm{gr}$. gave $1.0267 \mathrm{gr}$. $\mathrm{CO}^{2}$ and 0.3572 gr. HO.

IV. $0.6467 \mathrm{gr}$. gave $0.4708 \mathrm{gr}$. ammonio-chloride of platinum.

V. 31.925 grs. gave, by the method already described, $1.9510 \mathrm{gr}$. hulls.

VI. $0.7232 \mathrm{gr}$, of the hulls dried at $100^{\circ} \mathrm{C}$. gave 0.0135 gr. ashes.

These correspond in 100 parts to-

* Their analysis gave-

Ann. de Ch. et de Phys. vi. 385.

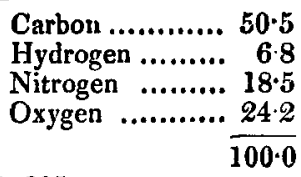


I.

Carbon ..... 45.32

Hydrogen ... 6.42

Nitrogen ..... 4.57

Ashes ...... 2.79

Water ..... 19.50

Hulls ...... 6.11

Ashes of hulls $\quad 1.86$

Estimated in 100 parts, the above determinations give the following numbers :-

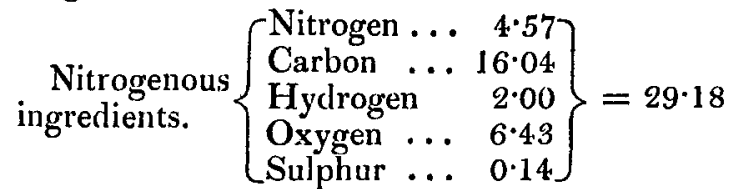

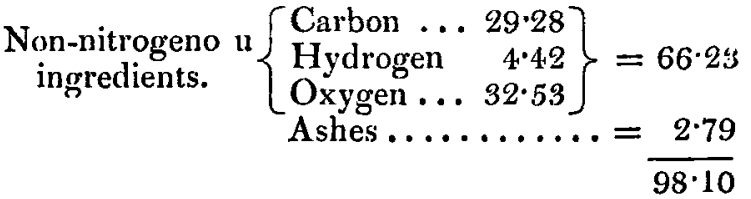

Legumine, according to Dumas' and Cahours' analysis, gave for the above determinations 101.06.

\begin{tabular}{|c|c|}
\hline \multirow{2}{*}{\multicolumn{2}{|c|}{$\begin{array}{cc} & \text { Dried at } 100^{\circ} \mathrm{C} \text {. } \\
\text { Nitrogenous inoredients } & 29 \cdot 18\end{array}$}} \\
\hline & $23 \cdot 49$ \\
\hline Inorganic ingredients $\ldots \quad 2 \times 79$ & $2 \cdot 24$ \\
\hline Woody fibre & $4 \cdot 83$ \\
\hline Starch, sugar, \&c. . . . . 62.03 & $51 \cdot 14$ \\
\hline Water ........... & 1950 \\
\hline $0.0 \Omega$ & 20 \\
\hline
\end{tabular}

Table Beans (Phaseolus vulgaris) from Vienna.

Berry bright, plump, and of less than medium size. 10 beans weighed $3 \cdot 1431 \mathrm{grms}$.

I. 2.9467 grs. lost at $100^{\circ}$ C. $0.3953 \mathrm{gr}$.

II. $2 \cdot 8422$ grs. of substance dried at $100^{\circ} \mathrm{C}$. gave $0 \cdot 1244$ gr. ashes.

III. $0.4648 \mathrm{gr}$, gave $0.7721 \mathrm{gr} . \mathrm{CO}^{2}$ and $0.2747 \mathrm{gr}$. HO.

IV. 0.4334. gr. gave $0.7126 \mathrm{gr} . \mathrm{CO}^{2}$ and $0.2617 \mathrm{gr}$. HO. num.

V. 0.9082 gr. gave $0 \cdot 64.57 \mathrm{gr}$. ammonio-chloride of plati-

VI. 13.6091 grs., by the method already described, gave $0.54 .61 \mathrm{gr}$. hulls.

VII. $0.5510 \mathrm{gr}$. hulls dried at $100^{\circ} \mathrm{C}$. gave $0.0212 \mathrm{gr}$, ashes.

These correspond in 100 parts to-

Phil. Mag. S. 3. Vol. 29. No. 195. Nov. 1846. 


\begin{tabular}{|c|c|}
\hline Carbon. .... & $\begin{array}{c}1 . \\
4.5 \cdot 30\end{array}$ \\
\hline Hydrogen ... & 6.56 \\
\hline Nitrogen ... & $4 \cdot 4 \cdot 7$ \\
\hline Ashes ...... & $4 \cdot 38$ \\
\hline Water ...... & $13 \cdot 4,1$ \\
\hline Hulls ...... & $4 \cdot 11$ \\
\hline Ashes of same & $3 \cdot 84$ \\
\hline Woody fibre & $3 \cdot 86$ \\
\hline
\end{tabular}

Estimated in 100 parts, the above determinations give the following numbers :-

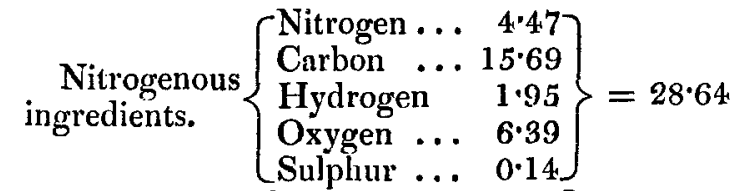

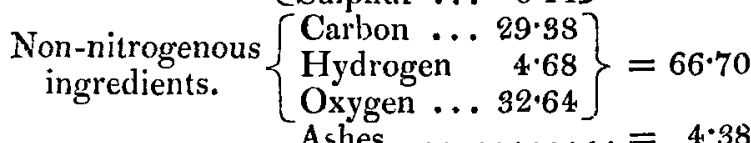

$$
\begin{aligned}
& \text { Ashes .......... }=\frac{4 \cdot 38}{99 \cdot 72}
\end{aligned}
$$

According to the analysis of legumine by Dumas and Cahours, the estimate gives 102.99.

$$
\begin{aligned}
& \text { Dried at } 100^{\circ} \mathrm{C} \text {. } \\
& \text { Nitrogenous ingredients }=28.54 \\
& \text { Inorganic ingredients } \ldots=4.38 \\
& \text { Woody fibre } \ldots \ldots \ldots=3.86 \\
& \text { Starch, sugar, \&c. } \ldots \ldots=63 \cdot 22 \\
& \text { Water } \\
& \overline{100 \cdot 00} \quad \frac{13 \cdot 41}{100 \cdot 00}
\end{aligned}
$$

\section{Large Field-beans (Vicia faba) from Giessen.}

White, plump and sound. 10 weigh 5.289 grms.

I. $7 \cdot 4054$ grs. lost at $100^{\circ} \mathrm{C} .1 \cdot 1705 \mathrm{gr}$.

II. $2.7950 \mathrm{grs}$. of substance dried at $100^{\circ} \mathrm{C}$. gave 0.1150 gr. ashes.

III. $0.4987 \mathrm{gr}$. gave $0.8255 \mathrm{gr}$. $\mathrm{CO}^{2}$ and $0.3053 \mathrm{gr}$. HO. num.

IV. $0.7238 \mathrm{gr}$. gave $0.5291 \mathrm{gr}$ ammonio-chloride of plati-

V. 44.5335 grs. gave, by the method already described, 1.9680 gr. hulls.

VI. $0.8335 \mathrm{gr}$. of the hulls dried at $100^{\circ} \mathrm{C}$. gave 0.0624 gr. ashes.

These, expressed in 100 parts, correspond to- 
Carbon .....445.18

Hydrogen ... 6.80

Nitrogen $\ldots .4 .59$

Ashes ..... 4:01

Water ..... 15.80

Hulls $\ldots . . .4 \cdot 44$

Hull-ashes ... 7०48

Woody fibre 4.09

Estimated in 100 parts, the above determinations give the following numbers:-

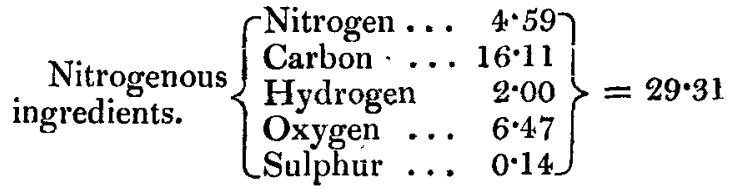

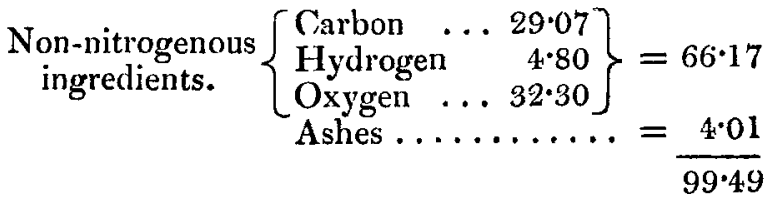

According to Dumas' and Cahours' analysis of legumine, the estimate from the above determinations gave $102 \cdot 73$.

$$
\begin{aligned}
& \text { Dried at } 100^{\circ} \mathrm{C} \text {. } \\
& \text { Nitrogenous ingredients }=29.31 \\
& \text { Inorganic ingredients }=4.01 \\
& \text { Woody fibre ....... }=4.09 \\
& \text { Starch, sugar, \&c. . . . . = } 62.59 \\
& \text { Fresh. } \\
& 24 \cdot 67 \\
& 3.37 \\
& 3 \cdot 44 \\
& 52 \cdot 72 \\
& \text { Water } \\
& 15 \cdot 80 \\
& 100 \cdot 00 \\
& \overline{100 \cdot 00}
\end{aligned}
$$

\section{Lentils (Ervum lens) from Vienna.}

Grains bright and sound.

I. 9.3074 grs. lost at $100^{\circ} \mathrm{C} .1 \cdot 2108 \mathrm{gr}$.

II. $2 \cdot 1669$ grs. pulverized lost at $100^{\circ} \mathrm{C} .0 \cdot 2820 \mathrm{gr}$.

III. $1 \cdot 4724 \mathrm{gr}$. of the substance dried at $100^{\circ} \mathrm{C}$. gave $0.0402 \mathrm{gr}$.

IV. $0.3511 \mathrm{gr}$. gave $0.5813 \mathrm{gr} . \mathrm{CO}^{2}$ and $0.2122 \mathrm{gr}$. HO.

V. 0.3863 gr. gave 0.6452 gr. $\mathrm{CO}^{2}$ and $0.2362 \mathrm{gr}$. HO.

VI. 0.6797 gr. gave $0.5198 \mathrm{gr}$. ammonio-chloride of platinum.

The above, expressed in 100 parts, correspond with-

$$
2 \mathrm{D} 2
$$


I. II.

Carbon ... 4:5.15

Hydrogen $\quad 6 \cdot 71$

Nitrogen $\quad 4: 77$

Ashes ... 2.60

Water ... 13.01
$4.5 \cdot 55$

$6 \cdot 79$

$13 \cdot 01$

Estimated in 100 parts, the above determinations give the following numbers:-

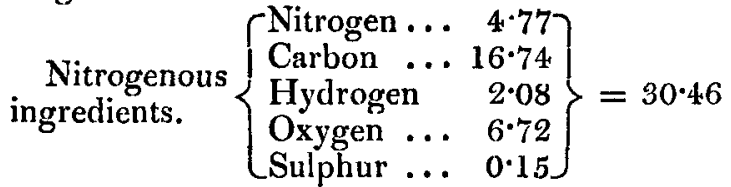

$$
\begin{aligned}
& \begin{array}{c}
\text { Non-nitrogenous } \\
\text { ingredients. }
\end{array} \quad\left\{\begin{array}{lr}
\text { Carbon .... } & 28 \cdot 61 \\
\text { Hydrogen } & 4 \cdot 67 \\
\text { Oxygen } \ldots . . & 31 \cdot 78
\end{array}\right\}=65 \cdot 06 \\
& \text { Ashes........... }=\frac{2 \cdot 60}{98 \cdot 12}
\end{aligned}
$$

According to Dumas' and Cahours' analysis of legumine, the estimate gave 101•34.

White Potatoes (Solanum tuberosum) from Giessen.

I. $1 \cdot 1455 \mathrm{gr}$. lost at $100^{\circ} \mathrm{C} .0 \cdot 8586 \mathrm{gr}$.

II. 3.2201 grs, substance dried at $100^{\circ} \mathrm{C}$. gave $0.1163 \mathrm{gr}$. ashes.

III. $0.5814 \mathrm{gr}$. gave $0.9351 \mathrm{gr}$. $\mathrm{CO}^{2}$ and $0.3139 \mathrm{gr}$. HO.

IV. $1 \cdot 1530 \mathrm{gr}$. gave $0 \cdot 2843 \mathrm{gr}$. ammonio-chloride of platinum.

These, expressed in 100 parts, give-

$$
\begin{array}{lr}
\text { Carbon .... } & 43 \cdot 86 \\
\text { Hyddrogen } & 6 \cdot 06 \\
\text { Nitrogen } & 1.56 \\
\text { Ashes ... } & 3 \cdot 61 \\
\text { Water ... } & 74 \cdot 95
\end{array}
$$

Estimated in 100 parts, the above determinations give the following numbers :-

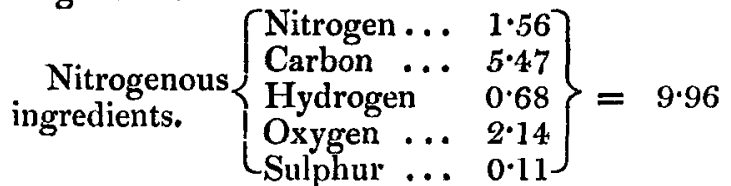

$$
\begin{aligned}
& \begin{array}{c}
\text { Non-nitrogenous } \\
\text { ingredients. }
\end{array}\left\{\begin{array}{lr}
\text { Carbon ... } & 38 \cdot 39 \\
\text { Hydrogen } & 5 \cdot 32 \\
\text { Oxygen ... } & 4 \cdot 2 \cdot 65
\end{array}\right\}=86 \cdot 36 \\
& \text { Ashes ........... }=\frac{3.61}{99.93}
\end{aligned}
$$


Blue Potatoes (Solanum tuberosum) from Giessen.

I. $2 \cdot 8369 \mathrm{grs}$. lost at $100^{\circ} \mathrm{C} .1 \cdot 9558 \mathrm{gr}$.

II. 3.6446 grs. of substance dried at $100^{\circ} \mathrm{C}$. gave 0.1226 gr. ashes.

III. $0.8315 \mathrm{gr}$. gave $1.3260 \mathrm{gr}$. $\mathrm{CO}^{2}$ and $0.4711 \mathrm{gr}$. HO.

IV. $0 \cdot 7625 \mathrm{gr}$. gave $1 \cdot 2030 \mathrm{gr}$. $\mathrm{CO}^{2}$ and $0.434 .5 \mathrm{gr}$. HO. num.

V. 1.3485 gr. gave 0.2587 gr. ammonio-chloride of plati-

Corresponding in 100 parts to-

\begin{tabular}{lrr} 
& \multicolumn{1}{c}{ I. } & \multicolumn{1}{c}{ II. } \\
Carbon ... & $43 \cdot 49$ & $43 \cdot 02$ \\
Hydrogen & $6 \cdot 29$ & $6 \cdot 33$ \\
Nitrogen & $1 \cdot 20$ & \\
Ashes ... & $3 \cdot 36$ & \\
Water ... & $68 \cdot 94$ &
\end{tabular}

Estimated in 100 parts, the above determinations lead to the following numbers:-

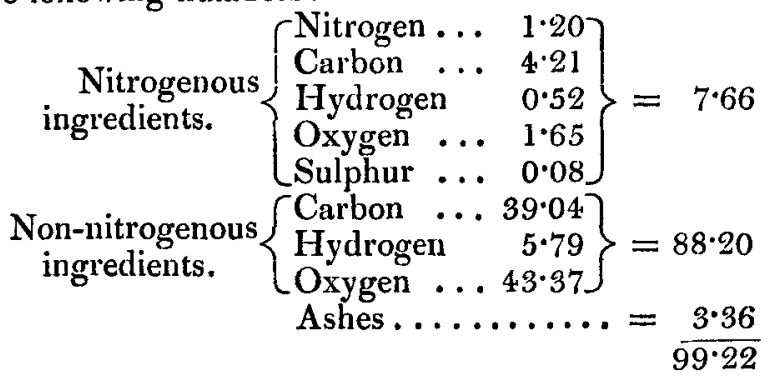

Carrots (Daucus carota) from Giessen.

I. 2.6735 grs. lost at $100^{\circ}$ C. 2.3021 gr.

II. $1.6379 \mathrm{gr}$. of substance dried at $100^{\circ} \mathrm{C}$. gave 0.0946 gr. ashes.

III. 0.6668 gr. gave $1.0597 \mathrm{gr}$. $\mathrm{CO}^{2}$ and $0.3736 \mathrm{gr}$. HO.

IV. 0.6797 gr. gave 0.1885 gr. ammonio-chloride of platinum.

V. $0.7043 \mathrm{gr}$, of the same gave $0.1790 \mathrm{gr}$. ammonio-chloride of platinum.

Expressed in 100 parts, these correspond to-

Carbon ... 4. 4.94

II.

Hydrogen 6.22

Nitrogen $1 \cdot 74$

Ashes ... 5.77

Water ... 86:10

Estimated in 100 parts, the above determinations give the following numbers :- 


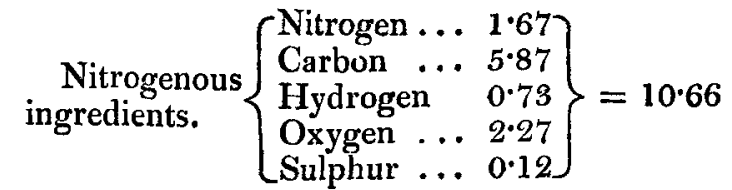

$$
\begin{aligned}
& \begin{array}{c}
\begin{array}{c}
\text { Non-nitrogenous } \\
\text { ingredients. }
\end{array} \\
\left.\begin{array}{l}
\text { Carbon ... 37.47 } \\
\text { Hydrogen } 5 \cdot 49 \\
\text { Oxygen. . } 41 \cdot 63
\end{array}\right\}=84.59 \\
\text { Ashes. ........... }
\end{array}
\end{aligned}
$$

Red Beets (Beta vulgaris rapacea) from Giessen.

1. $1.6173 \mathrm{gr}$. lost at $100^{\circ} \mathrm{C} .1 \cdot 3200 \mathrm{gr}$.

II. 2.3399 grs. of substance dried at $100^{\circ} \mathrm{C}$. gave 0.1505 gr. ashes.

III. $0.4875 \mathrm{gr}$. gave $0.7525 \mathrm{gr}$. $\mathrm{CO}^{2}$ and $0.2512 \mathrm{gr}$. HO. num.

IV. 0.5505 gr. gave 0.2152 gr. ammonio-chloride of plati-

Corresponding in 100 parts to-

Carbon ... 40.99

Hydrogen $\quad 5.72$

Nitrogen $\quad 2 \cdot 43$

Ashes ... 6.43

Water ...8 81.61

Estimated in 100 parts, the above determinations give the following numbers :-

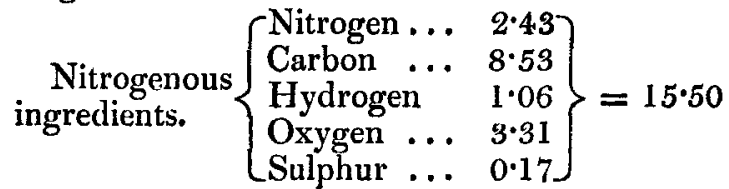

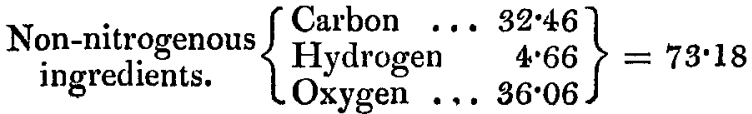

$$
\begin{aligned}
& \text { Ashes } \ldots \ldots \ldots \ldots=6.43 \\
& \overline{95 \cdot 11}
\end{aligned}
$$

Peligot found in this beet-

$$
\begin{array}{rcl}
10.6 & \text { per cent. sugar ; add to this } \\
2.8 & \ldots & \text { nitrogenous ingredients, } \\
1.1 & \ldots & \text { inorganic matter, } \\
81.6 & \ldots & \text { water, and there remain } \\
3.9 & \ldots & \text { woody fibre, \&c. in } \\
\frac{100.0}{100} \text { parts. } &
\end{array}
$$


Yellow French Beet (Beta cicla) from Giessen.

I. $1.8545 \mathrm{gr}$. lost at $100^{\circ}$ C. $1.5255 \mathrm{gr}$.

II. $2 \cdot 2840$ grs. of substance dried at $100^{\circ} \mathrm{C}$. gave 0.1148 gr. ashes.

III. $0.4530 \mathrm{gr}$. gave $0.6853 \mathrm{gr}$. $\mathrm{CO}^{2}$ and $0.2587 \mathrm{gr}$. HO.

IV. 0.4057 gr. gave $0.6165 \mathrm{gr}$. $\mathrm{CO}^{2}$ and $0.2202 \mathrm{gr}$. HO. num.

V. 0.5660 gr. gave $0.1635 \mathrm{gr}$. ammonio-chloride of plati-

Corresponding in 100 parts to-

I.

Carbon ... 41.25

Hydrogen $\quad 6.34$

Nitrogen 1.81

Ashes ... 5.02

Water ... 82.25
II.

$41 \cdot 45$

$6 \cdot 03$

Estimated in 100 parts, the above determinations give the following numbers:-

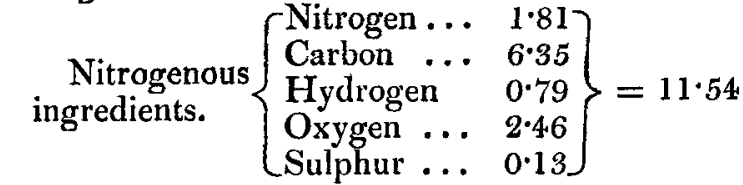

$$
\begin{aligned}
& \begin{array}{c}
\text { Non-nitrogenous } \\
\text { ingredients. }
\end{array}\left\{\begin{array}{l}
\text { Carbon ... } 34 \cdot 74 \\
\text { Hydrogen } \\
\text { Oxygen ... } 38 \cdot 60
\end{array}\right\}=78 \cdot 49 \\
& \text { Ashes .......... }=\frac{5.02}{99 \cdot 05}
\end{aligned}
$$

Ruta Baga (Brassica napo-brassica) from Giessen.

I. 2.2981 grs. lost at $100^{\circ}$ C. $1.9139 \mathrm{gr}$.

II. 2.5171 grs. of substance dried at $100^{\circ} \mathrm{C}$. gave $0 \cdot 1010$ gr. ashes.

III. $0.6762 \mathrm{gr}$. gave $1.1226 \mathrm{gr}$. $\mathrm{CO}^{2}$ and $0.3665 \mathrm{gr}$. $\mathrm{HO}$.

IV. 0.6122 gr. gave $1.0180 \mathrm{gr}$. $\mathrm{CO}^{2}$ and $0.3306 \mathrm{gr}$. HO.

V. 0.7625 gr. gave 0.1765 gr. ammonio-chloride of platinum.

Corresponding in 100 parts to-

I.

Carbon ... 45.27

Hydrogen 6.02

Nitrogen $1 \cdot 45$

Ashes ... $4 \cdot 10$

Water ... 83.28
II.

$45 \cdot 35$

$6 \cdot 00$

Estimated in 100 parts, the above determinations give the following numbers :- 
Nitrogenous $\left\{\begin{array}{lll}\text { Nitrogen... } & 1 \cdot 45 \\ \text { Carbon ... } & 5 \cdot 09 \\ \text { ingredients. } & \text { Hydrogen } & 0 \cdot 63 \\ \text { Oxygen ... } & 1 \cdot 97 \\ \text { Sulphur ... } & 0 \cdot 10\end{array}\right\}=9 \cdot 24$

$\begin{aligned} & \text { Non-nitrogenous } \\ & \text { ingredients. }\end{aligned}\left\{\begin{array}{lr}\text { Carbon ... } & 40 \cdot 23 \\ \text { Hydrogen } & 5 \cdot 38 \\ \text { Oxygen ... } & 44 \cdot 71\end{array}\right\}=90.32$

Ashes

$$
=\frac{4 \cdot 01}{103 \cdot 57}
$$

White Turnips (Brassica rapa) from Giessen.

I. $0.874 .2 \mathrm{gr}$. lost at $100^{\circ} \mathrm{C} .0 .7674 \mathrm{gr}$.

II. $1.7487 \mathrm{gr}$. of substance dried at $100^{\circ} \mathrm{C}$. gave 0.1229 gr. ashes.

III. $0.4376 \mathrm{gr}$ gave $0.6953 \mathrm{gr}$. $\mathrm{CO}^{2}$ and $0.2225 \mathrm{gr}$. $\mathrm{HO}$.

IV. 0.2831 gr. gave 0.4472 gr. $\mathrm{CO}^{2}$.

V. 0.5976 gr. gave 43.42 per cent. C. and 5.91 per cent. $H$. num.

VI. $0.7969 \mathrm{gr}$. gave $0.25 \& 3 \mathrm{gr}$. ammonio-chloride of plati-

Corresponding in 100 parts to-

I.

Carbon ... 43.33

Hydrogen 5.64

Nitrogen 1.98

Ashes ... 7.03

Water ... $87 \cdot 78$
II. 43.08

$6 \cdot 04$
III.

$48 \cdot 42$

$5 \cdot 91$

Estimated in 100 parts, the above determinations give the following numbers :-

$$
\begin{aligned}
& \text { Nitrogenous }\left\{\begin{array}{lll}
\text { Nitrogen... } & 1 \cdot 98 \\
\text { Carbon ... } & 6 \cdot 95 \\
\text { ingredients. } & \text { Jydrogen } & 0 \cdot 86 \\
\text { Oxygen ... } & 2 \cdot 69 \\
\text { Sulphur ... } & 0 \cdot 14
\end{array}\right\}=12 \cdot 62
\end{aligned}
$$

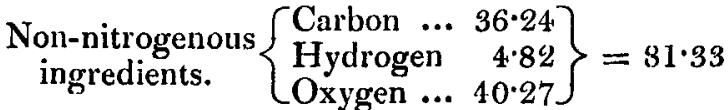

$$
\begin{aligned}
& \text { Ashes .............. }=7.02 \\
& \overline{100 \cdot 97}
\end{aligned}
$$

White Onions from Giessen.

I. 4.3169 grms. lost at $100^{\circ}$ C. 4.0478 grms.

II. $0.6775 \mathrm{gr}$. of substance dried at $100^{\circ} \mathrm{C}$. gave 0.0578 gr. ashes. 
III. $0.6726 \mathrm{gr}$, gave $0.1272 \mathrm{gr}$. ammonio-chloride of platinum.

These determinations correspond in 100 parts to-

$$
\begin{aligned}
& \text { Nitrogen... } \quad 1 \cdot 18 \\
& \text { Ashes ...... 8.53 } \\
& \begin{array}{lll}
\text { Water ... } & 93.78
\end{array}
\end{aligned}
$$

\begin{tabular}{|c|c|c|c|}
\hline Name. & $\begin{array}{l}\text { Undissolved re- } \\
\text { sidue in } 100 \\
\text { parts (hulls? } \\
\text { dried at } 100^{\circ} \mathrm{C} .\end{array}$ & $\begin{array}{l}\text { Ashes of } \\
\text { the hulls. }\end{array}$ & $\begin{array}{c}\text { Woody } \\
\text { fibre. }\end{array}$ \\
\hline Common Winter Barley ..... & $5 \cdot 40$ & $1 \cdot 90$ & $5 \cdot 30$ \\
\hline Tartarian Buckwheat .. & $22 \cdot 67$ & $0 \cdot 08$ & $22 \cdot 66$ \\
\hline Panicled Oats............ & $16 \cdot 66$ & $3 \cdot 35$ & $16 \cdot 10$ \\
\hline Table Peas ...... & $7 \cdot 65$ & $2 \cdot 47$ & $7 \cdot 47$ \\
\hline Field Peas ..... & $6 \cdot 11$ & 1.86 & $6 \cdot 00$ \\
\hline Table Beans ................................ & $4 \cdot 01$ & $3 \cdot 84$ & $3 \cdot 86$ \\
\hline Large Field-beans ......................... & $4 \cdot 41$ & $\mathbf{7} \cdot \mathbf{4 8}$ & $4 \cdot 09$ \\
\hline
\end{tabular}

Hulls.

\begin{tabular}{|c|c|c|c|c|}
\hline Name. & $\begin{array}{c}10 \text { grains } \\
\text { weighed in un- } \\
\text { dried condi- } \\
\text { tion. }\end{array}$ & $\begin{array}{l}\text { Relative weight } \\
\text { of the indivi- } \\
\text { dual grains, } \\
\text { Bush-rye }=1 \text {. }\end{array}$ & $\begin{array}{c}\text { Per cent. of } \\
\text { nitrogen in } \\
\text { undried con. } \\
\text { dition. }\end{array}$ & $\begin{array}{c}\text { Relative quan- } \\
\text { tity of nitro- } \\
\text { gen in the } \\
\text { individual } \\
\text { grains, } \\
\text { Bush-rye }=1 .\end{array}$ \\
\hline Bush-rye & grs. 0.1220 & 1 & $2 \cdot 39$ & 1 \\
\hline Rush-rye ... & $0 \cdot 1838$ & $1 \cdot 5$ & $2 \cdot 13$ & $1 \cdot 3$ \\
\hline Talavera Wheat & $0 \cdot 3606$ & 3 & $2 \cdot 19$ & $2 \cdot 7$ \\
\hline Whitington Wheat & $0 \cdot 4239$ & $3 \cdot 5$ & $2 \cdot 30$ & $2 \cdot 9$ \\
\hline Sandomierz Wheat ......... & $0: 3199$ & $2 \cdot 8$ & $2 \cdot 13$ & $2 \cdot 4$ \\
\hline Indian Corn .......... & $3 \cdot 5934$ & $29 \cdot 4$ & 1.95 & $24 \cdot 0$ \\
\hline Jerusalem Barley & 0.5312 & $4 \cdot 3$ & 1.92 & $3 \cdot 6$ \\
\hline Common Barley..... & $0 \cdot 3955$ & $3 \cdot 2$ & $2 \cdot 40$ & $2 \cdot 4$ \\
\hline Kamskatka Oats ... & $0 \cdot 3446$ & $2 \cdot 8$ & $2 \cdot 08$ & $2 \cdot 4$ \\
\hline Early-panicled Oats & $0 \cdot 3689$ & 3 & $2 \cdot 45$ & $3 \cdot 1$ \\
\hline Tartarian Buckwheat ...... & 0.2566 & $2 \cdot 1$ & $1 \cdot 33$ & $1 \cdot 2$ \\
\hline Table Peas .................... & $2 \cdot 6080$ & $21 \cdot 4$ & $\mathbf{3} \cdot \mathbf{8 3}$ & $34 \cdot 3$ \\
\hline Field Peas .. & 1.9828 & $16 \cdot 3$ & $3 \cdot 68$ & $25 \cdot 1$ \\
\hline 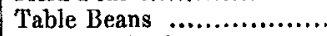 & $3 \cdot 1431$ & $25 \cdot 5$ & $3 \cdot 87$ & $4] \cdot 2$ \\
\hline Large White Beans ......... & $5 \cdot 2890$ & $43 \cdot 3$ & $\mathbf{3} \cdot 86$ & $69 \cdot 9$ \\
\hline
\end{tabular}

Relative worth of the individual grains according to weight and per-centage of nitrogen. 
Tabular Results of Analysis.

\begin{tabular}{|c|c|c|c|c|c|c|}
\hline Name. & $\begin{array}{l}\text { Nitro- } \\
\text { gen. }\end{array}$ & Carbon. & $\begin{array}{l}\text { Hydro- } \\
\text { gen. }\end{array}$ & Oxygen. & Sulphur. & Ashes. \\
\hline Wheat-flour from Vienna, No. $1 .$. & $3 \cdot 00$ & $45 \cdot 74$ & $6 \cdot 70$ & $43 \cdot 23$ & $0 \cdot 23$ & 0.70 \\
\hline Wheat-flour from Vienna, No. $2 \ldots$ & $2 \cdot 12$ & & & $44 \cdot 81$ & $0 \cdot 15$ & 0.66 \\
\hline Wheat-flour from Vienna, No. 3 .. & $3 \cdot 44$ & $46 \cdot 86$ & $6 \cdot 78$ & $42 \cdot 65$ & 25 & $1 \cdot 10$ \\
\hline Talavera Wheat from Hohenheim. & $2 \cdot 59$ & $44 \cdot 93$ & $6 \cdot 25$ & $43 \cdot 35$ & $0 \cdot 18$ & $2 \cdot 80$ \\
\hline Whit & $2 \cdot 68$ & $44 \cdot 42$ & $6 \cdot 82$ & $42 \cdot 56$ & $0 \cdot 19$ & $3 \cdot 13$ \\
\hline Sando & $2 \cdot 69$ & $44 \cdot 20$ & $6 \cdot 68$ & $42 \cdot 28$ & $0 \cdot 19$ & $2 \cdot 40$ \\
\hline Rye & 1.87 & $44 \cdot 37$ & $6 \cdot 65$ & $44 \cdot 55$ & $0 \cdot 13$ & $1 \cdot 33$ \\
\hline Rye- & $2 \cdot 93$ & $45 \cdot 19$ & $6 \cdot 56$ & $42 \cdot 77$ & $0 \cdot 21$ & 1.07 \\
\hline Bus & $\mathbf{2 \cdot 7}$ & $45 \cdot 52$ & & $43 \cdot 51$ & 15 & $0 \cdot 86$ \\
\hline & $2 \cdot 47$ & $45 \cdot 23$ & 6.57 & $43 \cdot 98$ & $\cdot 18$ & $\mathbf{2 \cdot 3 7}$ \\
\hline Pol & $2 \cdot 14$ & $45 \cdot 04$ & $6 \cdot 60$ & $44 \cdot 62$ & $0 \cdot 15$ & $0 \cdot 86$ \\
\hline Indis & $2 \cdot 30$ & $45 \cdot 45$ & $6 \cdot 61$ & $44 \cdot 66$ & $0 \cdot 16$ & 1.92 \\
\hline essen & $2 \cdot 07$ & $44 \cdot 54$ & 6.72 & $44 \cdot 24$ & $0 \cdot 15$ & 2.01 \\
\hline Shenheim & $2 \cdot 31$ & $45 \cdot 50$ & 7 & $44 \cdot 68$ & $0 \cdot 16$ & $2 \cdot 84$ \\
\hline $\left.\begin{array}{r}\text { Common Winter Barley from } \\
\text { Hohenheim } \ldots . . . \ldots \ldots \ldots \ldots \ldots \ldots\end{array}\right\}$ & $2 \cdot 79$ & $45 \cdot 22$ & $6 \cdot 99$ & $42 \div 6$ & 0.20 & 5.52 \\
\hline Kamskatka Oats from Hohenheirm & $2 \cdot 39$ & $46 \cdot 50$ & $6 \cdot 64$ & $\mathbf{4 5 \cdot 5 9}$ & $0 \cdot 17$ & $3 \cdot 26$ \\
\hline 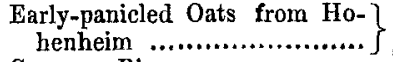 & $2 \cdot 82$ & $46 \cdot 66$ & $\cdot 74$ & $4 \cdot 68$ & $0 \cdot 20$ & $4 \cdot 14$ \\
\hline Comn & $1 \cdot 16$ & $44 \cdot 61$ & 3 & $46 \cdot 62$ & 08 & 36 \\
\hline Buck & $1 \cdot 08$ & $44 \cdot 27$ & & 50 & 07 & 09 \\
\hline $\begin{array}{l}\text { Tartarian Buckwheat from Ho } \\
\text { henheim }\end{array}$ & $1 \cdot 56$ & $45 \cdot 42$ & $6 \cdot 45$ & 46.50 & +11 & 30 \\
\hline Table Peas from Vienna ............ & 4 & 2 & $6 \cdot 73$ & 22 & 4 & $3 \cdot 18$ \\
\hline & $4 \cdot 5$ & 45 & 2 & 38.75 & $0 \cdot 1_{4}$ & 79 \\
\hline Tabl & $4 \cdot 47$ & $45 \cdot 07$ & $6 \cdot 63$ & $38 \cdot 73$ & $0 \cdot 14$ & 38 \\
\hline om Giessen & 4.59 & & 6. & 38 & $0 \cdot 14$ & 01 \\
\hline & 4.77 & 45 & 6.75 & & 0.15 & 60 \\
\hline & $1 \cdot 56$ & & 600 & & $0 \cdot 14$ & $3 \cdot 61$ \\
\hline Blt & $1 \cdot 20$ & $43 \cdot 25$ & $6 \cdot 31$ & $45 \cdot 00$ & 0.08 & 36 \\
\hline $\mathrm{C}_{2}$ & $1 \cdot 67$ & & & & $0 \cdot 12$ & $5 \cdot 77$ \\
\hline & $2 \cdot 43$ & 40 & $5 \cdot 7$ & $39 \cdot 37$ & $0 \cdot 17$ & $6 \cdot 43$ \\
\hline Rute & $1 \cdot 81$ & & & & $0 \cdot 13$ & $5 \cdot 02$ \\
\hline Yellow French & $1 \cdot 45$ & 45 & $6 \cdot 6$ & & $0 \cdot 10$ & $4 \cdot 01$ \\
\hline Whit & 1.98 & $43 \cdot 19$ & $5 \cdot 68$ & $42 \cdot 96$ & $0 \cdot 14$ & $7 \cdot 02$ \\
\hline White Onions from Giessen ....... & & & & & & \\
\hline
\end{tabular}

Table for the Nitrogenous Ingredients.

\begin{tabular}{|c|c|c|c|}
\hline \multirow{2}{*}{ Name. } & \multicolumn{2}{|c|}{ Nitrogenous ingredients. } & \multirow{2}{*}{ Water. } \\
\hline & $\begin{array}{l}\text { Dried at } \\
100^{\circ} \mathrm{C} .\end{array}$ & $\begin{array}{l}\text { In fresh con- } \\
\text { dition. }\end{array}$ & \\
\hline Wheat-flour from Vienna, No. 1 ........... & $19 \cdot 16$ & $16 \cdot 51$ & $13 \cdot 85$ \\
\hline Wheat-flour from Vienna, No. 2 .. & $13 \cdot 54$ & $11 \cdot 69$ & $13 \cdot 65$ \\
\hline Wheat-flour from Vienna, No. 3 ............. & $21 \cdot 97$ & $19 \cdot 17$ & $12 \cdot 73$ \\
\hline Talavera Wheat from Hohenheim & $.16 \cdot 54$ & $13 \cdot 98$ & 15.43 \\
\hline Whitington Wheat from Hohenheim ..... & $17 \cdot 11$ & 14.72 & $13 \cdot 93$ \\
\hline Sandomierz Wheat from Hohenheim ...... & $17 \cdot 18$ & $14 \cdot 5 \overline{1}$ & $15 \cdot 48$ \\
\hline Rye-flour from Vienna, No. 1 ............. & $11 \cdot 94$ & $10 \cdot 34$ & $13 \cdot \% 8$ \\
\hline Rye-flour from Vienna, No.2 $\ldots$ & $18 \cdot 71$ & 1596 & $14 \cdot 68$ \\
\hline Bush-rye from Hohenheim ........ & $17 \cdot 75$ & $15 \cdot 27$ & $13 \cdot 94$ \\
\hline Rush-rye from Hohenheim ...... & $15 \cdot 77$ & $13 \cdot 59$ & $13 \cdot 82$ \\
\hline Polenta Meal from Vienna ...... & $13 \cdot 66$ & 11.53 & $13 \cdot 36$ \\
\hline Indian Corn from Hohenheim .......... & $14 \cdot 65$ & $12 \cdot 48$ & $14: 96$ \\
\hline
\end{tabular}


Table for the Nitrogenous Ingredients (continued).

\begin{tabular}{|c|c|c|c|}
\hline \multirow[b]{2}{*}{ Name, } & \multicolumn{2}{|c|}{ Nitrogenous ingredients. } & \multirow[b]{2}{*}{ Water. } \\
\hline & $\begin{array}{l}\text { Dried at } \\
100^{\circ} \mathrm{C} .\end{array}$ & $\begin{array}{l}\text { In fresh con- } \\
\text { dition. }\end{array}$ & \\
\hline Triticum monococcum from Giessen & $13 \cdot 22$ & $11 \cdot 30$ & $14 \cdot 40$ \\
\hline Jerusalem Barley from Hohenheim.. & $14 \cdot 74$ & $12 \cdot 26$ & 16.79 \\
\hline Common Barley from Hohenheim .......... & $17 \cdot 81$ & $15 \cdot 35$ & $13 \cdot 80$ \\
\hline Kamskatka Oats from Hohenheim .......... & $15 \cdot 26$ & $13 \cdot 32$ & 12.71 \\
\hline Early-panicled Oats from Hohenheim... & $18 \cdot 00$ & $15 \cdot 67$ & $12 \cdot 94$ \\
\hline The same without hulls $\ldots . . . \ldots \ldots \ldots . . . . .$. & $21 \cdot 57$ & $18 \cdot 78$ & $12 \cdot 94$ \\
\hline Common Rice $\ldots \ldots \ldots \ldots \ldots \ldots \ldots \ldots \ldots . . . . . . . . .$. & $7 \cdot 40$ & $6 \cdot 27$ & $15 \cdot 14$ \\
\hline Buckwheat Meal from Vienna ..... & 6.89 & $5 \cdot 84$ & $15 \cdot 12$ \\
\hline Tartarian Buckwheat from Hohenheim. & $9 \cdot 96$ & $7 \cdot 94$ & $14 \cdot 19$ \\
\hline Table Peas from Vienna ..................... & $28 \cdot 02$ & $24 \cdot 41$ & $13 \times 43$ \\
\hline Field Peas from Giessen ....... & $29 \cdot 18$ & $23 \cdot 49$ & $19 \cdot 50$ \\
\hline Table Beans from Vienna ............ & $28 \cdot 54$ & $\mathbf{2 4 \cdot 7 4}$ & $13 \cdot 41$ \\
\hline Large White Beans from Giessen............ & $29 \cdot 31$ & $24 \cdot 67$ & $15 \cdot 80$ \\
\hline 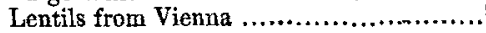 & $30 \cdot 46$ & 26.59 & $13 \cdot 01$ \\
\hline White Potatoes from Giessen .............. & $9 \cdot 96$ & $2 \cdot 49$ & 74.95 \\
\hline Blue Potatoes from Giessen.................. & $7 \cdot 66$ & $2 \cdot 37$ & $68 \cdot 94$ \\
\hline Carrots from Giessen $\ldots \ldots \ldots \ldots \ldots \ldots \ldots \ldots \ldots \ldots$ & $10 \cdot 66$ & $\mathbf{1} \cdot 48$ & $86 \cdot 10$ \\
\hline Red Beets from Giessen .................. & $15 \cdot 50$ & $\mathbf{2} \cdot \mathbf{8 3}$ & $81 \cdot 61$ \\
\hline Ruta Baga from Giessen ............... & $11 \cdot 56$ & 2.04 & $82 \cdot 25$ \\
\hline Yellow French Beet from Giessen & $9 \cdot 25$ & $\mathrm{I} \cdot \mathbf{5 4}$ & $83 \cdot 28$ \\
\hline White Turnips from Giessen ......... & $12 \cdot 64$ & 1.54 & 87.78 \\
\hline White Onions from Giessen .......... & $7 \cdot 53$ & $0 \cdot 46$ & 93.78 \\
\hline
\end{tabular}

Tabular Arrangement of Nutrition Values in Equivalents. Wheat at 100.

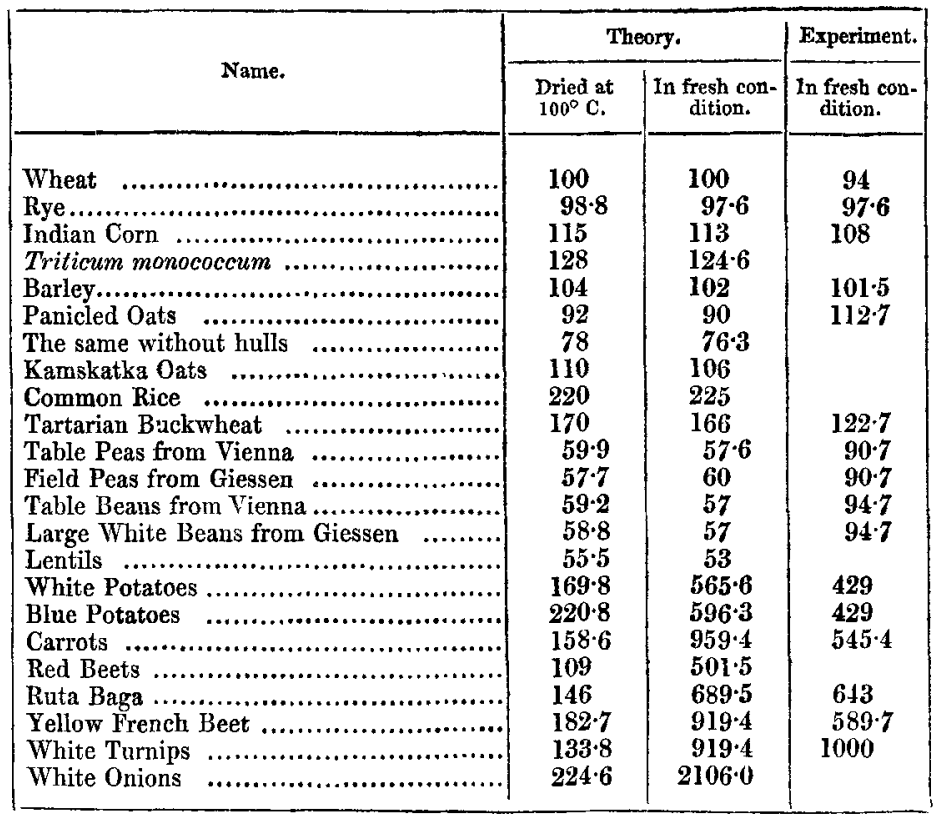


The last column in the preceding table contains the average results of experiments made to ascertain the practical equivalents, as they are given in Boussingault (Landwirthschaft, Germ. ed. p. 292-295). One of the results with wheat was neglected, its variation from the others being so great

By comparing the results of the above investigation with each other, and with those previously known, the following conclusions have been arrived at:-

That the same species of cereal grain grown on different soils may yield unequal per-centages of nitrogen.

That one-seventh of fresh ripe cereal grains is moisture, which may be expelled at a temperature of $100^{\circ} \mathrm{C} .\left(212^{\circ} \mathrm{F}\right.$.).

That wheat- and rye-flours, which to the eye and sense of feeling are undistinguishable from each other, may differ by from one to three-tenths of their whole quantity of nitrogen.

That root-crops grown on different soils may yield unequal per-centages of nitrogen.

That the per-centage of moisture in edible roots of the same species is, in the fresh condition, a constant quantity.

That beets, carrots and turnips, have a larger per-centage of moisture than potatoes.

That the nutritive values of peas, beans and lentils, correspond with each other.

That more aliment is contained in a given weight of peas, beans or lentils, than in an equal weight of any other kind of vegetable food analysed.

That in several of the grains and roots analysed there are organic bodies beside those identical in composition with gluten and starch.

That the ashes of carrots, beets, turnips and potatoes, as Prof. von Liebig has already remarked, contain carbonates.

That iron is present in the ashes of all the grains and roots analysed.

That the differences between the theoretical equivalents, as estimated from the per-centages of nitrogen and those ascertained by the experiments of stock-growers, and particularly the differences between the results of the differem stockgrowers, may be attributed to the following reasons:-

Because the per-centages of nitrogen and carbon in fodder grown on different soils are unequal.

Because the prominent test has been the increase or diminution in weight of the animal fed. Increase in weight may arise from secretion of fat, derived from the sugar and starch of the plants. Diminution in weight may follow unusual activity, increasing the consumption of fat already present.

Because the experiments, in but few instances, were under- 
taken with substances whose per-centages of water and nitrogen had been ascertained.

Because theoretical equivalents have been employed in conditions unequally suited to digestion. The same food, coarse or fine, fresh or prepared for easy digestion, yields unequal measures of nutrition.

Because the conditions, whether exposed to the open air or protected in stalls, whether subjected to labour of uniform severity, or allowed the free range of pastures, have not been made alike.

Finally, because, as above intimated, some animals by nature differ greatly from others, in the facility with which fat and muscle are developed, even when the circumstances are precisely the same.

LVIII. Microscopic Observations on the Perforation of the Capillaries by the Corpuscles of the Blood, and on the Origin of Mucus and Pus-globules. By Augustus Walle r, M.D.*

\section{[Continued from p. 287.]}

T $\mathrm{N}$ the preceding Number of this Journal I have already given 1 a brief outline of some observations respecting the manner in which the white corpuscles of the blood are seen to leave the interior of the capillary vessels. As this subject is intimately connected with the formation or origin of the globules found in mucus and purulent matter, it deserves a close and strict investigation.

In the first place, we must bear in mind that the blood consists of transparent fluid parts containing two kinds of particles in suspension; the one of a red colour and a flattened disc-like shape, and the other nearly transparent and spherical. The examination of the tongue of the frog, or of any other transparent part of a living animal, shows these globules and corpuscles generally circulating together through the capillaries.

The examination of mucus and purulent matter shows that they contain great quantities of globules, which are exactly similar to the spherical corpuscles found in the blood. In all these instances these particles are of a spherical form, transparent or whitish, granulated, and about the size of $\frac{1}{10} \frac{\text { th }}{0}$ of a millimetre. In acetic acid they contract, in the alkalies they are quickly broken up, in water they swell and are disaggregated into separate minute granules.

The corpuscles of blood in the mammiferæ and in the frog are found to be of about the same size as in man.

In proof of what I have stated respecting the similarity of

* Communicated by the Author. 